TRANSACTIONS OF THE

AMERICAN MATHEMATICAL SOCIETY

Volume 350, Number 7, July 1998, Pages 2903-2924

S $0002-9947(98) 02081-9$

\title{
PROJECTIVE THREEFOLDS ON WHICH SL(2) ACTS WITH 2-DIMENSIONAL GENERAL ORBITS
}

\author{
T. NAKANO
}

\begin{abstract}
The birational geometry of projective threefolds on which SL(2) acts with 2-dimensional general orbits is studied from the viewpoint of the minimal model theory of projective threefolds. These threefolds are closely related to the minimal rational threefolds classified by Enriques, Fano and Umemura. The main results are (i) the $\mathbf{S L}(2)$-birational classification of such threefolds and (ii) the classification of relatively minimal models in the fixed point free cases.
\end{abstract}

\section{INTRODUCTION}

Regular SL(2)-actions on algebraic varieties were studied from diverse points of view by several authors (see, for instance, [B], [Du1], [Du2], [LV], [P]). In this paper, we study the projective threefolds on which SL(2) acts regularly with 2-dimensional general orbits in terms of the minimal model theory of projective threefolds (= Mori theory, cf. $[\mathrm{CKM}])$.

Our main results are (i) the SL(2)-birational classification of such threefolds (Theorem 1.4) and (ii) the classification of relatively minimal models in the fixed point free cases (Theorems 4.1 and 4.2).

Projective threefolds with regular $\mathbf{S L}(2)$-action are closely related to the grouptheoretically minimal rational threefolds. Here a smooth rational projective variety $X$ of dimension $n$ is called group-theoretically minimal (abbreviated as g.m. in the following) if the connected component $\operatorname{Aut}^{0}(X)$ of the automorphism group of $X$ containing the identity is maximal in the Cremona group $\mathrm{Cr}_{n}$ of $n$ variables up to conjugation. Let us discuss this point in some detail, which is the main motivation for this study. In a series of papers [U1]-[U4], [Mu], Umemura, partly joint with Mukai, classified all the maximal connected algebraic subgroups of $\mathrm{Cr}_{3}$ up to conjugation, giving a rigorous proof to the works of Enriques and Fano $[\mathrm{EF}],[\mathrm{F}]$. Further, he studied and clarified the birational geometry of g.m. rational threefolds.

We note that, in dimension 2, g.m. rational surfaces are all toric and they coincide with the geometrically minimal ones (namely, those containing no exceptional curves of the first kind). However, in dimension 3, there exist g.m. rational threefolds $X$ whose $\operatorname{Aut}^{0}(X)$ is of rank 1 and 2 so that these are not toric (cf. [De]). Thus, toric threefolds do not cover all the g.m. rational threefolds.

From the viewpoint of group actions, we sought for a class of varieties which contains all the g.m. rational varieties, and observed that, for any g.m. rational

Received by the editors July 20, 1996.

1991 Mathematics Subject Classification. Primary 14L30; Secondary 14E30.

Key words and phrases. SL(2)-action, projective threefolds, minimal models. 
variety $X$ of dimension 2 and 3, there exists a simple algebraic group $G$ such that $G$ acts on $X$ regularly and nontrivially. Equivalently, $\operatorname{Aut}^{0}(X)$ is an unsolvable group for any g.m. rational variety $X$ at least in dimension 2 and 3 . Therefore we decided to study projective varieties with unsolvable $\operatorname{Aut}^{0}(\cdot)$. Let $\mathcal{U S}(n)$ be the set of all isomorphism classes of smooth projective $n$-folds with unsolvable Aut ${ }^{0}(\cdot)$. For the classification of $\mathcal{U S}(n)$ in low dimension $(n=1,2,3)$, see Proposition A in Appendix. We here note that projective threefolds on which $\mathbf{S L}(2)$ acts with 2 -dimensional general orbits are the last class in $\mathcal{U S}(3)$ whose structure is unknown.

This paper is organized as follows. In Section 1 we determine the $\mathbf{S L}(2)$ birational structure of projective threefolds on which $\mathbf{S L}(2)$ acts with 2-dimensional general orbits (Theorem 1.4). It turns out that $X$ is $\mathbf{S L}(2)$-birationally trivial except in one case. We also determine when $X$ has no fixed points (Theorem 1.5) in this section. From Section 2 to the end of this paper, we concentrate on the fixed point free cases. In Section 2 we study the contraction map of the extremal rays in the sense of [Mo]. In Section 3 we construct explicitly some examples of relatively minimal models (minimal in the geometric sense) and study their elementary transformations. Finally in Section 4 the classification of relatively minimal models in the fixed point free cases is done (Theorems 4.1 and 4.2). We note that our proofs for Theorems 4.1 and 4.2 consist in the description of contraction maps of extremal rays and the elementary transformations studied in Sections 2 and 3, respectively. In the Appendix we review the classification of $\mathcal{U S}(n)$ for $n=1,2,3$. The case where $X$ admits fixed points is more complicated and will be discussed in a forthcoming paper.

Notations and conventions. (1) In this paper, algebraic varieties and algebraic groups are defined over the field $\mathbf{C}$ of complex numbers.

(2) An algebraic $n$-fold is an irreducible, reduced algebraic variety of dimension $n$.

(3) If an algebraic group $H$ acts regularly on a variety $X$, we say that $X$ is an $H$-variety. In this note, when we call $X$ an $H$-variety, we assume that $H$ acts on $X$ non-trivially unless otherwise stated. For a point $P \in X$, we denote by $O(P)$ and $H_{P}$ the orbit through $P$ and the isotropy group of $P$, respectively.

(4) Assume that $f: X \rightarrow Y$ is a rational map between $H$-varieties. We say that $f$ is $H$-rational if $f$ is $H$-equivariant where defined.

(5) Throughout this paper, $G$ denotes the special linear group $\mathbf{S L}(2, \mathbf{C})$ of degree 2 .

(6) When a group $M$ acts on an abelian group $N$ from the right, the semi-direct product of $M$ and $N$ is denoted by $M \ltimes N$, where multiplication is defined by $\left(m_{1}, n_{1}\right)\left(m_{2}, n_{2}\right)=\left(m_{1} m_{2}, n_{1}^{m_{2}} n_{2}\right)$.

\section{1. $G$-Birational Classification}

1.1. Let $G=\mathbf{S L}(2, \mathbf{C})$ be the special linear group of degree 2. We review the classification of smooth projective $G$-curves and $G$-surfaces for later use.

Proposition 1.1. (1) Let $C$ be a smooth projective $G$-curve. Then $C$ is isomorphic to the projective line $\mathbf{P}^{1}$.

(2) Let $S$ be a smooth projective $G$-surface. Then $S$ is isomorphic to one of the following; $\mathbf{P}^{2}, \mathbf{P}^{1} \times \mathbf{P}^{1}, \Sigma_{n}(n \geq 1), C \times \mathbf{P}^{1}$, where $\Sigma_{n}:=\mathbf{P}\left(\mathcal{O}_{\mathbf{P}^{1}} \oplus \mathcal{O}_{\mathbf{P}^{1}}(-n)\right)$ is the Hirzebruch surface of degree $n$ and $C$ is a smooth projective curve. 
Proof. The assertion (1) is obvious. For the proof of (2), see [Mab, Theorem 5.1].

We describe briefly the $G$-actions and orbit structures of $G$-varieties in Proposition 1.1. Let $V_{n}(n \geq 1)$ be the irreducible $G$-module of complex dimension $n$, which is unique up to isomorphisms. $V_{n}$ may be identified with the vector space of binary forms of degree $n-1$ with the natural $G$-action.

The $G$-action on $\mathbf{P}^{1}$ is given by identifying $\mathbf{P}^{1}$ with the projectivization $\mathbf{P}\left(V_{2}\right)$ of $V_{2} . \mathbf{P}\left(V_{2}\right)$ is a homogeneous space of $G$, and we have $\mathbf{P}\left(V_{2}\right) \cong G / B$, where $B$ is a Borel subgroup of $G ; B:=\left\{\left(\begin{array}{cc}a & b \\ 0 & a^{-1}\end{array}\right) \mid a \in \mathbf{C}^{\times}, b \in \mathbf{C}\right\}$.

We have two different $G$-actions on $\mathbf{P}^{2} ; \mathbf{P}^{2} \cong \mathbf{P}\left(V_{3}\right)$ consists of two orbits: a smooth conic $Q \cong G / B$ and $\mathbf{P}^{2}-Q \cong G / N(T)$. Here $N(T):=T \cup\left(\begin{array}{cc}0 & 1 \\ -1 & 0\end{array}\right) \cdot T$, where $T$ is the torus; $T:=\left\{\left(\begin{array}{cc}a & 0 \\ 0 & a^{-1}\end{array}\right) \mid a \in \mathbf{C}^{\times}\right\} . \mathbf{P}^{2} \cong \mathbf{P}\left(V_{2} \oplus V_{1}\right)$ consists of three orbits: a fixed point $P$, a line $\cong G / B$ and a 2 -dimensional orbit $\cong G / F_{1}$, where $F_{n}:=\left\{\left(\begin{array}{cc}\zeta & b \\ 0 & \zeta^{-1}\end{array}\right) \mid \zeta^{n}=1, b \in \mathbf{C}\right\}$ for a positive integer $n$.

$\mathbf{P}\left(V_{2}\right) \times \mathbf{P}\left(V_{2}\right) \cong \mathbf{P}^{1} \times \mathbf{P}^{1}$ consists of two orbits: the diagonal $D \cong G / B$ and $\mathbf{P}^{1} \times \mathbf{P}^{1}-D \cong G / T$.

As for $\Sigma_{n}(n>0)$, we note that $\mathcal{O}_{\mathbf{P}^{1}}(-1)$ has a natural $G$-linearization induced by the inclusion $\mathcal{O}_{\mathbf{P}^{1}}(-1) \subset \mathbf{P}\left(V_{2}\right) \times V_{2}$, and hence so does $\mathcal{O}_{\mathbf{P}^{1}}(-n)=\mathcal{O}_{\mathbf{P}^{1}}(-1)^{\otimes n}$. Thus $\Sigma_{n}$ has a natural $G$-action induced by that on $\mathcal{O}_{\mathbf{P}^{1}} \oplus \mathcal{O}_{\mathbf{P}^{1}}(-n)$. We denote by $\pi_{n}: \Sigma_{n} \rightarrow \mathbf{P}^{1}$ the natural projection. $\Sigma_{n}$ consists of three orbits: $C_{\infty} \cong G / B$, $C_{0} \cong G / B$ and $\Sigma_{n}^{\prime}:=\Sigma_{n}-\left(C_{\infty} \cup C_{0}\right) \cong G / F_{n}$, where $C_{\infty}$ (resp. $\left.C_{0}\right)$ is the section of $\pi_{n}$ defined by the projection $\mathcal{O}_{\mathbf{P}^{1}} \oplus \mathcal{O}_{\mathbf{P}^{1}}(-n) \rightarrow \mathcal{O}_{\mathbf{P}^{1}}(-n)\left(\right.$ resp. $\mathcal{O}_{\mathbf{P}^{1}} \oplus \mathcal{O}_{\mathbf{P}^{1}}(-n) \rightarrow$ $\left.\mathcal{O}_{\mathbf{P}^{1}}\right)$.

Finally $G$ acts on $C \times \mathbf{P}^{1}$ by the product action, where $G$ acts on $C$ trivially and on $\mathbf{P}^{1}$ via $\mathbf{P}^{1} \cong \mathbf{P}\left(V_{2}\right)$.

Now we prepare a simple lemma which is used in the proofs of Theorem 1.4, Proposition 4.3 and Lemma 4.4 .

Lemma 1.2. For any integer $d \geq 2$, there exists a cyclic $G$-covering $g_{d}: \Sigma_{n} \rightarrow \Sigma_{d n}$ of degree $d$ such that the restriction $\left.g_{d}\right|_{\Sigma_{n}^{\prime}}: \Sigma_{n}^{\prime} \rightarrow \Sigma_{n d}^{\prime}$ coincides with the natural surjection $G / F_{n} \rightarrow G / F_{n d}$.

Proof. The natural surjection $G / F_{n} \rightarrow G / F_{n d}$ is a cyclic $G$-covering of degree $d$. Let $g_{d}: \Sigma_{n} \rightarrow \Sigma_{n d}$ be the $G$-rational map induced by $\Sigma_{n}^{\prime} \cong G / F_{n} \rightarrow G / F_{n d} \cong \Sigma_{n d}^{\prime}$. Then $g_{d}$ is an everywhere defined $G$-morphism and defines the desired $G$-cyclic covering.

1.2. Let $X$ be a smooth projective $G$-threefold and $m$ the maximal dimension of all orbits of $X$. Suppose $m=2$. Then there exist a non-empty $G$-stable open subset $X_{0} \subset X$ and a closed subgroup $H$ of $G$ of dimension 1 such that, for any point $x \in X_{0}$, the isotropy group $G_{x}$ of $x$ is conjugate to $H$ in $G$ by [Du2, Theorem 3]. We call an orbit isomorphic to $G / H$ a general orbit of $X$. (For the cases $m=1,3$, see Appendix.) Let $H$ be a 1-dimensional closed subgroup of $G=\mathbf{S L}(2)$. Then it is well-known that $H$ is conjugate to either $F_{n}(n \geq 1), T$ or $N(T)$.

Definition 1.3. Let $H$ be a 1-dimensional closed subgroup of $G$.

(1) We denote by $\mathcal{C}(H)$ the set of all $G$-isomorphism classes of smooth projective $G$-threefolds whose general orbit is isomorphic to $G / H$. 
(2) For $X \in \mathcal{C}(H)$, a smooth projective curve $C$ is called the invariant curve of $X$ if the rational function field $k(C)$ of $C$ is isomorphic to the $G$-invariant subfield $k(X)^{G}$ of $k(X)$.

1.3. We state and prove the $G$-birational classification of $X \in \mathcal{C}(H)$, where $H$ is a 1-dimensional closed subgroup of $G$. Let $C$ be a smooth projective curve with the trivial $G$-action and we denote by $S$ one of the $G$-surfaces described in Proposition 1.1 (2). We consider the product $G$-action on the product variety $S \times C$ and call this the $G$-trivial fibration over $C$. We also say that $X$ is $G$-birationally trivial if $X$ is $G$-birational to the $G$-trivial fibration.

Let $f: C^{\prime} \rightarrow C^{\prime \prime}$ be an étale double covering of smooth curves. $C^{\prime \prime}$ is isomorphic to $C^{\prime} /\langle\alpha\rangle$, where $\alpha$ is an involution of $C^{\prime}$. Let $\beta$ be an involution of $\mathbf{P}^{1} \times \mathbf{P}^{1} \times C^{\prime}$ defined by $\beta((P, Q, x))=(Q, P, \alpha(x))$, where $(P, Q, x) \in \mathbf{P}^{1} \times \mathbf{P}^{1} \times C^{\prime}$, and set $V_{f}:=\left(\mathbf{P}^{1} \times \mathbf{P}^{1}\right.$-diagonal $) \times C^{\prime} /\langle\beta\rangle$. Consider the standard $G$-action on $\mathbf{P}^{1} \times \mathbf{P}^{1} \cong$ $\mathbf{P}\left(V_{2}\right) \times \mathbf{P}\left(V_{2}\right)$ and the trivial $G$-action on $C^{\prime}$. Then the product $G$-action on $\mathbf{P}^{1} \times \mathbf{P}^{1} \times C^{\prime}$ commutes with $\beta$ and hence induces a $G$-action on $V_{f}$.

Theorem 1.4. Let $X$ be a smooth projective $G$-threefold with 2-dimensional general orbits.

(1) If $X \in \mathcal{C}\left(F_{n}\right)$ or $\mathcal{C}(N(T))$, then $X$ is $G$-birationally trivial.

(2) If $X \in \mathcal{C}(T)$, then $X$ is either $G$-birationally trivial or $G$-birational to $V_{f}$ for some $f$. Further, $V_{f}$ is not G-birationally trivial.

Proof. Step 1. By [Du2], there exist a non-empty open $G$-stable subset $V$ of $X$ and a 1-dimensional closed subgroup $H$ of $G$ such that, for any $x \in V, G_{x}$ is conjugate to $H$ and the quotient $p: V \rightarrow V / G$ exists. We complete $V / G$ to a smooth projective curve $C$ (with the trivial $G$-action) and denote by $\pi: X \rightarrow C$ the $G$-rational map induced by $p$. We resolve the indeterminacy locus of $\pi$ by $\sigma: \widetilde{X} \rightarrow X$ which is a composition of $G$-blowing-ups so that $\varphi:=\pi \circ \sigma$ is a $G$-morphism. We may assume that $\varphi^{-1}(x)$ is connected for any $x \in C$ by taking Stein factorization. Then there exists a non-empty open subset $W \subset C$ such that, for any $x \in W, \varphi^{-1}(x)$ is all isomorphic to one and only one $G$-surface $F$, where $F$ is either (i) $\Sigma_{n}(n \geq 1)$, $\mathbf{P}\left(V_{1} \oplus V_{2}\right) \cong \mathbf{P}^{2}$, (ii) $\mathbf{P}\left(V_{2}\right) \times \mathbf{P}\left(V_{2}\right) \cong \mathbf{P}^{1} \times \mathbf{P}^{1}$, or (iii) $\mathbf{P}\left(V_{3}\right) \cong \mathbf{P}^{2}$.

By $[\mathrm{FG}],\left.\varphi\right|_{\varphi^{-1}(W)}$ is analytically locally trivial. Namely, for any $x \in W$, there exists a complex analytic neighborhood $U_{x}$ in $W$ such that $\varphi^{-1}\left(U_{x}\right) \cong F \times U_{x}$. Hence we have a 1-parameter family of homomorphisms of Lie algebras $\rho_{y}: \operatorname{Lie}(G) \rightarrow$ $\operatorname{Lie}\left(\operatorname{Aut}^{0}(F)\right)\left(y \in U_{x}\right)$, where $\operatorname{Lie}(G)$ is the Lie algebra of $G$. Since $L=\operatorname{Lie}(G)$ is semi-simple, $H^{1}(L, \rho)=\{0\}$ for any finite-dimensional representation $\rho$ of $L$ and hence there exists a 1-parameter family of inner automorphisms $i_{y}$ of $\operatorname{Lie}\left(\operatorname{Aut}^{0}(F)\right)$ such that $\rho_{y}=i_{y} \circ \rho_{x}$ for all $y \in U_{x}$ (we shrink $U_{x}$ if necessary). See [H, Chap. D, Sec. 7,8$]$ for details. Hence we find that $\varphi^{-1}\left(U_{x}\right)$ is $G$-isomorphic to $F \times U_{x}$, where $G$ acts on $U_{x}$ trivially and on $F \times U_{x}$ by the product action.

Consider the contravariant function $\mathcal{F}:\{W$-schemes $\} \rightarrow$ ssets $\}$ defined by $\mathcal{F}(Z)$ $:=$ the set of all $G$-equivariant $Z$-isomorphisms between $\varphi^{-1}(W) \times_{W} Z$ and $F \times Z$, where $G$ acts on $Z$ trivially. If $Z=\operatorname{Spec}(A)$ is affine, then a $G$-equivariant $Z$ isomorphism between $\varphi^{-1}(W) \times_{W} Z$ and $F \times Z$ is described by a finite number of polynomial equations with coefficients in $A$. Thus $\mathcal{F}$ is of finite presentation (cf. [A, Corollary (1.6)]). Let $\mathcal{O}_{x}$ be the local ring of $W$ at $x$ and we denote by $\widehat{\mathcal{O}}_{x}$ (resp. $\widetilde{\mathcal{O}}_{x}$ ) the formal completion (resp. the Henzelization) of $\mathcal{O}_{x}$. Since $\varphi^{-1}(W) \times_{\operatorname{Spec} \mathcal{O}_{x}} \operatorname{Spec}\left(\widehat{\mathcal{O}}_{x}\right)$ is $G$-isomorphic to $F \times \operatorname{Spec}\left(\widehat{\mathcal{O}}_{x}\right)$ from the argument 
above, $\mathcal{F}\left(\operatorname{Spec}\left(\widehat{\mathcal{O}}_{x}\right)\right)$ is non-empty. By $[\mathrm{A}], \mathcal{F}\left(\operatorname{Spec}\left(\widetilde{\mathcal{O}}_{x}\right)\right)$ is also non-empty, and we conclude that there exists an étale neighborhood $s: \widetilde{U} \rightarrow W$ of $x$ such that $\varphi^{-1}(W) \times_{W} \widetilde{U}$ is $G$-isomorphic to $F \times \widetilde{U}$. We may assume that $s$ is finite étale by shrinking $W$. By taking the Galois closure of $s$, we find that $\varphi^{-1}(W)$ is $G$-birational to the quotient $F \times \widetilde{U} / K$, where $K$ is a finite subgroup of the group $\operatorname{Aut}^{G}(F \times \widetilde{U})$ of the $G$-automorphisms of $F \times \widetilde{U}$.

Step 2. Consider the following exact sequence of groups (we set $D:=\widetilde{U}$ ):

$$
1 \rightarrow \operatorname{Aut}_{D}^{G}(F \times D) \stackrel{\alpha}{\longrightarrow} \operatorname{Aut}^{G}(F \times D) \stackrel{\beta}{\longrightarrow} \operatorname{Aut}(D) \rightarrow 1,
$$

where $\operatorname{Aut}_{D}^{G}(F \times D)$ is the group of $D$-automorphisms of $F \times D$ which are $G$ equivariant. We have a canonical section $\gamma$ of $\beta$ defined by $\gamma(\sigma):=i d_{F} \times \sigma$ and hence $\operatorname{Aut}^{G}(F \times D) \cong$ Aut $D \ltimes \operatorname{Aut}_{D}^{G}(F \times D)$.

(i) Assume $F=\Sigma_{n}$. The case $F=\mathbf{P}\left(V_{1} \oplus V_{2}\right)$ is handled in the same way as the case $F=\Sigma_{1}$. We first note that $\operatorname{Aut}_{D}^{G}\left(\Sigma_{n} \times D\right)$ is the set of all 1-parameter families of $G$-automorphisms of $\Sigma_{n}$ parametrized by $D$, and we have

$$
\operatorname{Aut}^{G}\left(\Sigma_{n}\right) \cong \operatorname{Aut}^{G}\left(G / F_{n}\right) \cong N\left(F_{n}\right) / F_{n} \cong B / F_{n} \cong \mathbf{C}^{\times},
$$

where $N\left(F_{n}\right)$ is the normalizer of $F_{n}$ in $G$. Hence we find that $\operatorname{Aut}_{D}^{G}\left(\Sigma_{n} \times D\right) \cong$ $\mathcal{O}(D)^{\times}$. Let $K \subset \operatorname{Aut}^{G}\left(\Sigma_{n} \times D\right)$ be the finite subgroup such that $X$ is $G$-birational to $\Sigma_{n} \times D / K$ and set $K^{\prime}:=\beta(K), K^{\prime \prime}:=\operatorname{ker}\left(\left.\beta\right|_{K}: K \rightarrow K^{\prime}\right)$. Since $K^{\prime \prime}$ is a finite subgroup of $\operatorname{Aut}_{D}^{G}\left(\Sigma_{n} \times D\right) \cong \mathcal{O}(D)^{\times}, K^{\prime \prime}$ is isomorphic to a cyclic group $C_{d}$ of order $d$ consisting of the $d$-th roots of unity. By taking a quotient by $K^{\prime \prime}$ first, we may replace $\Sigma_{n}$ by $\Sigma_{d n}$ and assume that $\left.\beta\right|_{K}: K \rightarrow K^{\prime}$ is an isomorphism (cf. Lemma 1.1). Now, we show that $K$ is conjugate to $K^{\prime}$ in $\operatorname{Aut}^{G}\left(\Sigma_{d n} \times D\right)$ (we identify $K^{\prime}$ with $\gamma\left(K^{\prime}\right) \subset$ Aut $^{G}\left(\Sigma_{d n} \times D\right)$ ), concluding that $X$ is $G$-birational to $\Sigma_{d n} \times D^{\prime}$, where $D^{\prime}:=D / K^{\prime}$. Let $k(D)^{\times}$be the multiplicative group of the rational function field of $D$ and consider the map $F: K^{\prime} \rightarrow k(D)^{\times}$defined by $\sigma \in K^{\prime} \Leftrightarrow(\sigma, F(\sigma)) \in K \subset \operatorname{Aut}(D) \ltimes k(D)^{\times}$. Since

$$
(\sigma, F(\sigma)) \cdot(\tau, F(\tau))=\left(\sigma \tau, F(\sigma)^{\tau} F(\tau)\right)=(\sigma \tau, F(\sigma \tau)),
$$

we have $F(\sigma \tau)=F(\sigma)^{\tau} F(\tau)$ for any $\sigma, \tau \in K^{\prime}$. Hence $F$ is a 1-cocycle $\epsilon$ $C^{1}\left(K^{\prime}, k(D)^{\times}\right)$in the sense of Galois cohomology. By Hilbert's Theorem 90, there exists $g \in k(D)^{\times}$such that $F(\sigma)=g \cdot\left(g^{-1}\right)^{\sigma}$ for every $\sigma \in K^{\prime}$. Hence we have $\left(i d_{D}, g\right)^{-1} K^{\prime}\left(i d_{D}, g\right)=K$ by replacing $D$ by a Zariski-open subset if necessary and thus $X$ is $G$-birational to $\Sigma_{d n} \times D^{\prime}$, where $D^{\prime}=D / K^{\prime}$.

(ii) Assume $F=\mathbf{P}\left(V_{2}\right) \times \mathbf{P}\left(V_{2}\right)$. Then we have the following exact sequence:

$$
1 \rightarrow \operatorname{Aut}_{D}^{G}\left(\mathbf{P}^{1} \times \mathbf{P}^{1} \times D\right) \rightarrow \operatorname{Aut}^{G}\left(\mathbf{P}^{1} \times \mathbf{P}^{1} \times D\right) \rightarrow \operatorname{Aut}(D) \rightarrow 1 .
$$

Since $\operatorname{Aut}_{D}^{G}\left(\mathbf{P}^{1} \times \mathbf{P}^{1} \times D\right) \cong N(T) / T \simeq C_{2}$, we get $\operatorname{Aut}^{G}\left(\mathbf{P}^{1} \times \mathbf{P}^{1} \times D\right) \cong C_{2} \times$ $\operatorname{Aut}(D)$ (direct product). If $K \subset \operatorname{Aut}(D)$, then $X$ is $G$-birational to $\mathbf{P}^{1} \times \mathbf{P}^{1} \times D^{\prime}$, where $D^{\prime}=D / K$. If $K$ contains an element $(-1, \sigma) \in C_{2} \times \operatorname{Aut}(D)$, then we have

$$
1 \rightarrow K_{2} \rightarrow K \stackrel{\left.p_{2}\right|_{K}}{\longrightarrow} C_{2} \rightarrow 1,
$$

where $p_{2}: \operatorname{Aut}(D) \times C_{2} \rightarrow C_{2}$ is the projection to the second factor and $K_{2}=$ $\operatorname{ker}\left(p_{2}\right)$. By taking quotient by $K_{2}$ first, we may assume that $\left.p_{2}\right|_{K}: K \rightarrow C_{2}$ is an isomorphism. Then $K=\langle(-1, \sigma)\rangle$, where $\sigma$ is an involution of $D$. Since $-1 \in C_{2}$ corresponds to the involution $(P, Q) \mapsto(Q, P)$ of $\mathbf{P}^{1} \times \mathbf{P}^{1}$, we find that $X$ is $G$ birational to $V_{f}$ for some $f$. 
We show that $V_{f}$ is not $G$-birationally trivial, where $f: C^{\prime} \rightarrow C^{\prime \prime}$ is an étale morphism of degree 2 and $C^{\prime \prime} \cong C^{\prime} /\langle\alpha\rangle$. Suppose that $V_{f}$ is $G$-birationally trivial. Then $V_{f}$ is $G$-birational to $\mathbf{P}\left(V_{2}\right) \times \mathbf{P}\left(V_{2}\right) \times C^{\prime \prime}$ since $C^{\prime \prime}$ is the invariant curve of $V_{f}$. Hence $V_{f}$ is $G$-isomorphic to the quotient of the fibered product $\left(\mathbf{P}^{1} \times \mathbf{P}^{1} \times\right.$

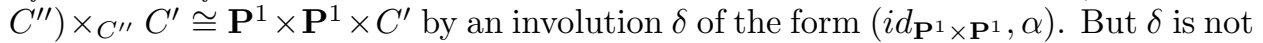
conjugate to the involution $\beta$ (see the construction of $V_{f}$ ) in $\operatorname{Aut}^{G}\left(\mathbf{P}^{1} \times \mathbf{P}^{1} \times C^{\prime}\right)$, a contradiction.

(iii) Assume $F=\mathbf{P}\left(V_{3}\right)$. The we have $\operatorname{Aut}_{D}^{G}\left(\mathbf{P}^{2} \times D\right) \cong N(T) / N(T) \cong\{1\}$ and hence $X$ is $G$-birational to the $G$-trivial fibration.

1.4. We next determine when a given $G$-action admits a fixed point. The result is as follows.

Theorem 1.5. If $X \in \mathcal{C}(H)$ has a fixed point, then $H=F_{1}$ or $T$.

Proof. Let $P \in X$ be a $G$-fixed point and consider the induced linear representation of $G$ on the tangent space $T_{P}(X) \cong \mathbf{C}^{3}$ at $P$.

(1) Assume that $T_{P}(X)$ is $G$-isomorphic to $V_{3}$. With respect to a suitable base of $T_{P}(X), G$ acts on $T_{P}(X)$ by

$$
g \circ v=\left(\begin{array}{ccc}
a^{2} & a b & b^{2} \\
2 a c & a d+b c & 2 b d \\
c^{2} & c d & d^{2}
\end{array}\right)\left(\begin{array}{l}
x \\
y \\
z
\end{array}\right)
$$

where $g=\left(\begin{array}{ll}a & b \\ c & d\end{array}\right) \in G$ and $v=\left(\begin{array}{l}x \\ y \\ z\end{array}\right) \in \mathbf{C}^{3}$.

We set $X_{k}:=\left\{(x, y, z) \in \mathbf{C}^{3} \mid y^{2}-4 x z=k\right\}$ for $k \in \mathbf{C}$. The $G$-orbits decomposition of $T_{P}(X) \cong \mathbf{C}^{3}$ is given as follows:

$$
\mathbf{C}^{3}=\{(0,0,0)\} \cup\left(X_{0}-\{(0,0,0)\}\right) \cup\left(\bigcup_{k \in \mathbf{C}-\{0\}} X_{k}\right) .
$$

We have $X_{0}-\{(0,0,0)\} \cong G / F_{2}$ and $X_{k} \cong G / T(k \neq 0)$. By Luna's étale slice theorem [L], there exist an affine open $G$-stable neighborhood $U$ of $P$ and an étale $G$ morphism $\nu: U \rightarrow T_{P}(X)$. Hence we find that the general orbit of $U$ is isomorphic to $G / T$.

(2) Assume that $T_{P}(X) \cong V_{1} \oplus V_{2}$. Then the $G$-orbits decomposition of $T_{P}(X) \cong$ $\mathbf{C}^{3}$ is given as follows:

$$
\mathbf{C}^{3}=\{z \text {-axis }\} \cup\left(\bigcup_{k \in \mathbf{C}} Y_{k}\right),
$$

where $z$-axis is the fixed point locus, $Y_{k}=\left\{(x, y, k) \in \mathbf{C}^{3} \mid x, y \in \mathbf{C}\right\}-\{(0,0,0\} \cong$ $G / F_{1}$. Hence we find that the general orbit of $X$ is $G / F_{1}$ by the similar method as in $(1)$.

(3) Assume that $T_{P}(X) \cong V_{1}^{\oplus 3}$. Then this contradicts the assumption that the dimension of the general orbits of $X$ is 2 by the similar method as in (1), (2).

\section{Study of CONTRACTION MAP}

In this section, we study the contraction map $f_{R}: X \rightarrow Y$ of an extremal ray $R$ on $X \in \mathcal{C}(H)$, mainly for $H=F_{n}(n \geq 2)$, in the sense of [Mo]. We first note that $f_{R}$ is defined by a complete linear system $|m L|(m \gg 0)$ for a certain line bundle $L \in \operatorname{Pic}(X)$ (cf. [CKM, Lecture 5]), and hence a regular $G$-action is defined 
on $Y$ such that $f_{R}$ is $G$-equivariant by $[S$, Theorem 1.6]. We also note that there exists an extremal ray on $X \in \mathcal{C}(H)$ since $X$ is birationally unruled by Theorem 1.4 (actually ruled by [Mat, Th. 1]).

Lemma 2.1. Let $R$ be an extremal ray of $X \in \mathcal{C}(H)$ and $f_{R}: X \rightarrow Y$ a birational contraction of $R$ such that the exceptional set $E$ of $f_{R}$ is isomorphic to $\mathbf{P}^{1} \times \mathbf{P}^{1}$. Then

(1) If $\mathbf{P}^{1} \times \mathbf{P}^{1}$ is G-isomorphic to $\mathbf{P}\left(V_{2}\right) \times \mathbf{P}\left(V_{2}\right)$, then $X \in \mathcal{C}(T)$.

(2) If $\mathbf{P}^{1} \times \mathbf{P}^{1}$ is G-isomorphic to $\mathbf{P}\left(V_{2}\right) \times \mathbf{P}_{\mathrm{tr}}^{1}$, where $\mathbf{P}_{\mathrm{tr}}^{1} \cong \mathbf{P}\left(V_{1}^{\oplus 2}\right)$, then $X \in \mathcal{C}\left(F_{1}\right)$.

(3) The case where $G$ acts on $E \cong \mathbf{P}^{1} \times \mathbf{P}^{1}$ trivially does not occur.

Proof. (1) $E \cong \mathbf{P}\left(V_{2}\right) \times \mathbf{P}\left(V_{2}\right)$ has a 2-dimensional orbit isomorphic to $G / T$. Hence the general orbit $G / H$ deforms to $G / T$. Then we have $H=T$ by [Du1, 2. Main results].

(2) Set $f_{R}(E)=\{P\}$ and let $m_{P}$ be the maximal ideal of the local ring $\mathcal{O}_{Y, P}$. Then we have $m_{P} / m_{P}^{2} \cong \Gamma\left(\mathbf{P}^{1} \times \mathbf{P}^{1}, \mathcal{O}_{\mathbf{P}^{1} \times \mathbf{P}^{1}}(1,1)\right) \cong V_{2} \oplus V_{2}$. We identify $V_{2}$ with $\mathbf{C}^{2}$ equipped with the standard $G$-action. Let $f_{i} \in m_{P} / m_{P}^{2}(1 \leq i \leq 4)$ be the element corresponding to the standard basis of $V_{2} \oplus V_{2}$ through this isomorphism. Then there exist a $G$-stable affine open neighborhood $U$ of $P$ and $F_{i} \in \Gamma\left(U, \mathcal{O}_{Y}\right)$ $(1 \leq i \leq 4)$ such that (i) $F_{i}(P)=0$ and $F_{i}$ induces $f_{i}$ in $m_{P} / m_{P}^{2}$, (ii) the morphism $F: U \rightarrow \mathbf{C}^{4}$ defined by $x_{i}=F_{i}(Q)(Q \in U, 1 \leq i \leq 4)$ is a $G$-equivariant map to $V_{2} \oplus V_{2} \cong \mathbf{C}^{4}$ (cf. [Mu, Lemma (1.2.2)]). Then $\operatorname{Im}(F)$ is contained in $Q:=$ $\left\{x_{1} x_{4}-x_{2} x_{3}=0\right\}$ and $F: U \rightarrow Q$ is generically finite. It is easy to see that the general orbit of $Q$ is 2-dimensional and isomorphic to $G / F_{1}$. Hence $X \in \mathcal{C}\left(F_{1}\right)$.

(3) Arguing similarly as in (2), we define a $G$-equivariant morphism $h: U \rightarrow \mathbf{C}^{4}$, where $U$ is a $G$-stable open affine neighborhood of $P$ and the $G$-action on $\mathbf{C}^{4}$ is trivial. Since $h$ is generically finite onto $\operatorname{Im}(h)$, the $G$-action on $X$ is also trivial, contradiction.

Now we concentrate on $\mathcal{C}\left(F_{n}\right)(n \geq 2)$. We recall that $X \in \mathcal{C}\left(F_{n}\right)(n \geq 2)$ has no fixed points by Theorem 1.5 .

Proposition 2.2. Let $R$ be an extremal ray of $X \in \mathcal{C}\left(F_{n}\right)(n \geq 2)$ such that $f_{R}: X \rightarrow Y$ is birational. Then $Y$ is smooth and $f_{R}$ is a $G$-blowing-up of $Y$ along a curve $C \subset Y$ isomorphic to $\mathbf{P}^{1} \cong \mathbf{P}\left(V_{2}\right)$.

Proof. Assume that $Y$ has a singular point $P$ and set $E=f_{R}^{-1}(P)$. We have three cases by [Mo, Theorem (3.3)].

(i) Suppose that $E \cong \mathbf{P}^{2}$. Then $E$ is $G$-isomorphic to $\mathbf{P}\left(V_{3}\right)$ since $X$ is fixed point free. $\mathbf{P}^{2} \cong \mathbf{P}\left(V_{3}\right)$ has an open dense orbit isomorphic to $G / N(T)$. By the deformation theory [Du1], we conclude $X \in \mathcal{C}(T)$ or $\mathcal{C}(N(T))$, contradiction. Hence this case does not occur.

(ii) Suppose that $E \cong \mathbf{P}^{1} \times \mathbf{P}^{1}$. Since $X$ has no fixed points, $E$ is $G$-isomorphic to either $\mathbf{P}\left(V_{2}\right) \times \mathbf{P}\left(V_{2}\right)$ or $\mathbf{P}\left(V_{2}\right) \times \mathbf{P}_{\text {tr }}^{1}$. Then we have $X \in \mathcal{C}(T)$ or $X \in \mathcal{C}\left(F_{1}\right)$ by Lemma 2.1, contradiction.

(iii) Suppose that $E$ is isomorphic to the singular quadric cone in $\mathbf{P}^{3}$. Since the singular point of $E$ is a fixed point of $X$, we get a contradiction.

Hence we conclude that $Y$ is smooth. Since $Y$ has no fixed points, $f_{R}$ is a blowing-up of $Y$ along a smooth irreducible curve $C$. We note that $C$ is a closed 1-dimensional $G$-orbit and hence $G$-isomorphic to $\mathbf{P}^{1} \cong \mathbf{P}\left(V_{2}\right)$. 
Remark. In the case of $X \in \mathcal{C}(N(T)), X$ may obtain a singular point by a birational contraction. Indeed, set $X=W_{2}=\mathbf{P}\left(\mathcal{O}_{\mathbf{P}^{2}} \oplus \mathcal{O}_{\mathbf{P}^{2}}(-2)\right)$ (see Subsection 3.4 for the notations). Then any line $l$ in the infinite section $S_{\infty} \cong \mathbf{P}^{2}$ is an extremal rational curve. Let $R=\mathbf{R}_{+}[l]$ be the extremal ray spanned by $l$. Then $f_{R}: W_{2} \rightarrow Y$ is the blowing-down of $S_{\infty}$ and $Y$ is isomorphic to the Veronese cone in $\mathbf{P}\left(V_{5} \oplus V_{1}\right) \cong \mathbf{P}^{5}$. See the remark after Proposition 2.7 for more details.

Proposition 2.3. Let $R$ be an extremal ray of $X \in \mathcal{C}\left(F_{n}\right)(n \geq 2)$ such that $f_{R}: X \rightarrow Y$ is a conic bundle. Then the following assertions hold.

(1) $Y$ is $G$-isomorphic to either $\Sigma_{m}(n \mid m)$ or $C \times \mathbf{P}\left(V_{2}\right)$, where $G$ acts on $C$ trivially.

(2) Every fiber of $f_{R}$ is smooth (and hence isomorphic to $\mathbf{P}^{1}$ ).

Proof. (1) By [Mo], $Y$ is a smooth projective surface with a non-trivial $G$-action. Hence $Y$ is $G$-isomorphic to one of $G$-surfaces in Proposition $1.1(2)$. Now $\mathbf{P}\left(V_{3}\right)$ $\left(\mathbf{P}\left(V_{2} \oplus V_{1}\right), \mathbf{P}\left(V_{2}\right) \times \mathbf{P}\left(V_{2}\right)\right.$ resp. $)$ has a 2-dimensional orbit of the form $G / N(T)$ $\left(G / F_{1}, G / T\right.$ resp.), and hence $Y$ cannot be these three $G$-surfaces since $G / F_{n}$ ( $n \geq$ 2) does not map onto $G / H$, where $H=T, N(T), F_{1}$.

(2) This follows from [U4, Lemma (1.13)].

Proposition 2.4. Let $X \in \mathcal{C}\left(F_{n}\right)(n \geq 2)$ and $R$ an extremal ray of $X$. Suppose that $f_{R}: X \rightarrow Y$ is a del Pezzo fiber space over a smooth projective curve $Y$. Then $Y \cong \mathbf{P}\left(V_{2}\right)$ and $f_{R}$ is a $\mathbf{P}^{2}$-bundle over $\mathbf{P}\left(V_{2}\right)$.

For the proof of this proposition, we need a lemma. Let $B=\left\{\left(\begin{array}{cc}a & b \\ 0 & a^{-1}\end{array}\right) \mid a \in\right.$ $\mathbf{C}^{\times}, b \in \mathbf{C}$ \} be the Borel subgroup of $G$. We have an isomorphism $B \cong \mathbf{C}^{\times} \ltimes \mathbf{C}$, where $\mathbf{C}^{\times}$acts on $\mathbf{C}$ by $A^{a}=a^{-2} A,(a, A) \in \mathbf{C}^{\times} \times \mathbf{C}$. Consider the semi-direct product $B_{2}:=\mathbf{C}^{\times} \ltimes_{2} \mathbf{C}$, where $\mathbf{C}^{\times}$acts on $\mathbf{C}$ by $A^{a}=a^{-4} A,(a, A) \in \mathbf{C}^{\times} \times \mathbf{C}$. Then $B_{2}$ is a double covering of $B$ via the map $B_{2} \ni(a, A) \mapsto\left(a^{2}, A\right) \in B$.

Lemma 2.5. (1) Let $\varphi: B_{2} \rightarrow \mathbf{S L}(2)$ be an algebraic homomorphism. Then $\varphi$ is one of the following up to conjugation:

(i) $\varphi(a, A)=\left(\begin{array}{cc}a^{2} & a^{2} A \\ 0 & a^{-2}\end{array}\right)$.

(ii) $\varphi(a, A)=\left(\begin{array}{cc}a^{m} & 0 \\ 0 & a^{-m}\end{array}\right)(m=0,1,2, \ldots)$.

(2) Suppose that $B$ acts on $\mathbf{P}^{1} \times \mathbf{P}^{1}$ in such a way that the general orbit is 1-dimensional. Then this $B$-action is induced by one of the following $\psi: B_{2} \rightarrow$ $\mathbf{S L}(2) \times \mathbf{S L}(2)$ defined by

(i) $\psi(a, A)=\left(\left(\begin{array}{cc}a^{2} & a^{2} A \\ 0 & a^{-2}\end{array}\right),\left(\begin{array}{ll}1 & 0 \\ 0 & 1\end{array}\right)\right)$.

(ii) $\psi(a, A)=\left(\left(\begin{array}{cc}1 & 0 \\ 0 & 1\end{array}\right),\left(\begin{array}{cc}a^{2} & a^{2} A \\ 0 & a^{-2}\end{array}\right)\right)$.

(iii) $\psi(a, A)=\left(\left(\begin{array}{cc}a^{m} & 0 \\ 0 & a^{-m}\end{array}\right),\left(\begin{array}{cc}a^{n} & 0 \\ 0 & a^{-n}\end{array}\right)\right)(m, n=0,1,2, \ldots,(m, n) \neq(0,0))$. up to conjugation.

Proof. (1) We may assume $\operatorname{Im} \varphi \subset B$ and $\varphi: \mathbf{C}^{\times} \ltimes_{2} \mathbf{C} \rightarrow \mathbf{C}^{\times} \ltimes \mathbf{C}$. Set $\varphi_{1}=\left.\varphi\right|_{\mathbf{C}^{\times}}$ and $\varphi_{2}=\left.\phi\right|_{\mathbf{C}}$. Then we may assume $\varphi_{1}(a)=\left(a^{m}, 0\right)$ and $\varphi_{2}(A)=(1, k A)$ for some $k \in \mathbf{C}$. Hence $\varphi(a, A)$ must be of the form $\varphi_{1}(a) \varphi_{2}(A)=\left(a^{m}, k A\right)$. Since $\varphi((a, A)(b, B))=\left(a^{m} b^{m}, k\left(b^{-4} A+B\right)\right)$ and $\varphi(a, A) \varphi(b, B)=\left(a^{m} b^{m}, k b^{-2 m} A+k B\right)$, we have $k A b^{-2 m}=k A b^{-4}$, concluding $k=0$ or $m=2$. We note that, if $k \neq 0$, then we may assume $k=1$ up to conjugation.

(2) This follows immediately from (1).

Proof of Proposition 2.4. We first note that $G$ acts on $Y$ non-trivially. Indeed, assume that $G$ acts on $Y$ trivially. Then the general fiber of $f:=f_{R}$ is a smooth 
del Pezzo surface which is $G$-stable. This is absurd since $\Sigma_{n}(n \geq 2)$ is not a del Pezzo surface. Hence $G$ acts on $Y$ non-trivially and we conclude that $Y \cong \mathbf{P}\left(V_{2}\right)$. Since $\mathbf{P}\left(V_{2}\right) \cong G / B$ is $G$-homogeneous, every fiber of $f$ is a smooth del Pezzo surface, which is either a blowing-up of $\mathbf{P}^{2}$ at $r$ points $(1 \leq r \leq 8), \mathbf{P}^{1} \times \mathbf{P}^{1}$ or $\mathbf{P}^{2}$. Now, fix a point $P=(1,0) \in \mathbf{P}^{1}$ of which the isotropy group is $B$. Then the fiber $f^{-1}(P)$ is a $B$-surface and we claim that $f^{-1}(P) \cong \mathbf{P}^{2}$. Indeed, assume that $f^{-1}(P)$ is a blowing-up of $\mathbf{P}^{2}$ with center $\left\{P_{1}, \ldots, P_{r}\right\}(1 \leq r \leq 8)$. Let $E \subset f^{-1}(P)$ be an exceptional curve over some $P_{i}$ and $l \subset f^{-1}(P)$ the proper transform of a line in $\mathbf{P}^{2}$ which does not go through $\left\{P_{1}, \ldots, P_{r}\right\}$. Then $E$ is $B$ stable and we set $D=G \circ E:=\{g \circ x \mid g \in G, x \in E\}$, which is an irreducible divisor in $X$. Then $(D, E)=\left(D, D, f^{-1}(P)\right)=(E, E)_{f^{-1}(P)}=-1$ and $(D, l)=0$, where $(\cdot, \cdot)$ (resp. $\left.(\cdot, \cdot)_{f^{-1}(P)}\right)$ is the intersection paring on $X$ (resp. $\left.f^{-1}(P)\right)$. This is a contradiction since $E \equiv x l$ for some $x>0$ (三 stands for the numerical equivalence) by the definition of contraction maps.

Next, assume that $f^{-1}(P) \cong \mathbf{P}^{1} \times \mathbf{P}^{1}$. Then, the $B$-action on $\mathbf{P}^{1} \times \mathbf{P}^{1}$ is induced by one of $\psi$ 's in Lemma 2.5 (2) since the general orbit of $X$ is 2-dimensional. Set $l_{1}=\{(1,0)\} \times \mathbf{P}^{1}$ and $l_{2}=\mathbf{P}^{1} \times\{(1,0)\}$. Then $l_{i}(i=1,2)$ is a $B$-stable line and we set $D_{1}=G \circ l$. We have $\left(D_{1}, l_{2}\right)=1$ and $\left(D_{1}, l_{1}\right)=0$, a contradiction. Thus we conclude $f^{-1}(P) \cong \mathbf{P}^{2}$ and $f: X \rightarrow \mathbf{P}^{1}$ is a $\mathbf{P}^{2}$-bundle.

Now we define a relatively minimal model in $\mathcal{C}(H)$ (minimal in a geometric sense).

Definition 2.6. We say that $X \in \mathcal{C}(H)$ is relatively minimal (or a relatively minimal model) if any $G$-birational morphism $f: X \rightarrow Y$ from $X$ to $Y \in \mathcal{C}(H)$ is an isomorphism (note that we are assuming $Y$ is smooth).

In the case $H=F_{n}(n \geq 2)$ or $N(T)$, we may say that $X \in \mathcal{C}(H)$ is relatively minimal if $X$ cannot be blown-down to some $Y \in \mathcal{C}(H)$. This follows from the following proposition.

Proposition 2.7. Let $X, Y \in \mathcal{C}(H)$, where $H=F_{n}(n \geq 2)$ or $N(T)$, and $f: X \rightarrow$ $Y$ a G-birational morphism. Then $f$ is a composition of finitely many blowing-ups along a closed 1-dimensional orbit isomorphic to $\mathbf{P}\left(V_{2}\right)$.

Proof. If $f$ is not an isomorphism, then there exists an extremal ray $R$ on $X$ such that $f$ factors through $f_{R}: X \rightarrow Z$ by [Mo, Corollary (3.41)]. Since $Y$ is fixed point free (Theorem 1.5), $Z$ is also fixed point free. Therefore $Z$ is smooth and $f_{R}$ is a $G$-equivariant blowing-up along a closed orbit $\cong \mathbf{P}\left(V_{2}\right)$. Repeating this process, we get the conclusion.

Remark. We have another way of defining relative minimality which is stronger than that in Definition 2.6, following the general theory of minimal models of projective threefolds (cf. [CKM, Lectures 1-16]). Namely, we say (provisionally) that $X \in$ $\mathcal{C}(H)$ is relatively minimal in Mori's sense if, for any extremal ray $R$ of $X, f_{R}$ is not birational. (In this case, it would be natural to work in the wider category of projective threefolds with only $\mathbf{Q}$-factorial terminal singularities.) Our justification for Definition 2.6 is as follows.

In the case of $\mathcal{C}\left(F_{n}\right)(n \geq 2)$, our definition of relative minimality coincides with that in Mori's sense by Proposition 2.2. In the case of $\mathcal{C}(N(T))$, we observe the following fact: 
Let $X \in \mathcal{C}(N(T))$ and suppose $f_{R}: X \rightarrow Y$ is a birational contraction. Then the following assertions hold;

(1) The contraction $f_{R}$ is either a blowing-up of smooth $Y$ along a closed 1dimensional orbit (type (a)), or a collapse of $E \cong \mathbf{P}^{2}$ with $\mathcal{O}_{E}(E) \cong \mathcal{O}_{\mathbf{P}^{2}}(-2)$ to a singular point of $Y$ (type (b)). Both types actually occur.

(2) If $R$ is an extremal ray such that $f_{R}$ is a birational contraction of type (b), then there exists another extremal ray $R^{\prime}$ such that $f_{R^{\prime}}$ is a birational contraction of type (a), except in the case of $X \cong W_{2}:=\mathbf{P}\left(\mathcal{O}_{\mathbf{P}^{2}} \oplus \mathcal{O}_{\mathbf{P}^{2}}(-2)\right)$.

This follows from Theorem 1.5, Lemma 2.1, and Theorem 4.2 in Section 4. Thus our definition coincides with that in Mori's sense except in the case $X \cong W_{2}$.

\section{Construction of minimal models}

In this section, we construct several types of examples of relatively minimal models and also discuss some properties of them, expecially elementary transformations.

3.1. $X_{n}(a, b) \in \mathcal{C}\left(F_{d}\right)$, where $d=\operatorname{gcd}(b, n)$. Let $\pi_{n}: \Sigma_{n} \rightarrow \mathbf{P}^{1}$ be the Hirzebruch surface of degree $n$ as usual. Consider a vector bundle $E_{n}(a, b)$ on $\Sigma_{n}$ defined by $E_{n}(a, b):=\left(\mathcal{O}_{\Sigma_{n}}(a) \otimes \pi_{n}^{*}\left(\mathcal{O}_{\mathbf{P}^{1}}(b)\right)\right) \oplus \mathcal{O}_{\Sigma_{n}}$, where $\mathcal{O}_{\Sigma_{n}}(a)=\mathcal{O}_{\Sigma_{n}}(1)^{\otimes a}$ and $\mathcal{O}_{\Sigma_{n}}(1)$ is the tautological line bundle on $\Sigma_{n}=\mathbf{P}\left(\mathcal{O}_{\mathbf{P}^{1}} \oplus \mathcal{O}_{\mathbf{P}^{1}}(-n)\right)$. Since a natural $G$-bundle structure on $E_{n}(a, b)$ is induced from the $G$-action on $\Sigma_{n}$, the induced $\mathbf{P}^{1}$-bundle $p_{n}: X_{n}(a, b):=\mathbf{P}\left(E_{n}(a, b)\right) \rightarrow \Sigma_{n}$ is a smooth projective $G$-threefold.

We set $D_{\infty}:=p_{n}^{-1}\left(C_{\infty}\right)$ and $D_{0}:=p_{n}^{-1}\left(C_{0}\right)$. Further, let $S_{1}$ (resp. $\left.S_{2}\right) \subset$ $X_{n}(a, b)$ be the section of $p_{n}$ defined by the projection $E_{n}(a, b) \rightarrow \mathcal{O}_{\Sigma_{n}}(a) \otimes$ $\pi_{n}^{*}\left(\mathcal{O}_{\mathbf{P}^{1}}(b)\right)\left(\right.$ resp. $\left.E_{n}(a, b) \rightarrow \mathcal{O}_{\Sigma_{n}}\right)$.

Lemma 3.1. (1) $S_{i}$ is a G-equivariant section of $p_{n}$ and isomorphic to $\Sigma_{n}$. $D_{\infty} \cong$ $\Sigma_{|b-n a|}$ and $D_{0} \cong \Sigma_{|b|}$.

(2) For any $x \in X_{n}(a, b)-\left(D_{\infty} \cup D_{0} \cup S_{1} \cup S_{2}\right)$, the orbit $O(x)$ through $x$ is isomorphic to $G / F_{d}$, where $d:=\operatorname{gcd}(b, n)$. In particular, $X_{n}(a, b) \in \mathcal{C}\left(F_{d}\right)$.

Proof. (1) The first assertion is clear. The second assertion follows by restricting $E_{n}(a, b)$ to $C_{0}$ and $C_{\infty}$ since $\mathcal{O}_{\Sigma_{n}}(1) \cong \mathcal{O}_{\Sigma_{n}}\left(C_{\infty}\right), \mathcal{O}_{\Sigma_{n}}\left(C_{0}\right) \cong \mathcal{O}_{\Sigma_{n}}\left(C_{\infty}\right) \otimes$ $\pi_{n}^{*}\left(\mathcal{O}_{\mathbf{P}^{1}}(n)\right)$ and $C_{\infty}^{2}=-n$.

(2) Take points $P=(1,0) \in \mathbf{P}^{1}$ and $Q=(x, y) \in \pi_{n}^{-1}(P) \cong \mathbf{P}^{1} \subset \Sigma_{n}, Q \neq$ $(1,0),(0,1)$. Then $g=\left(\begin{array}{cc}\alpha & \beta \\ 0 & \alpha^{-1}\end{array}\right) \in B=G_{P}$ acts on the fiber $\pi_{n}^{*}\left(\mathcal{O}_{\mathbf{P}^{1}}(1)\right)_{Q} \cong \mathbf{C}$ by multiplication by $\alpha^{-1}$. On the other hand, $G_{Q}=\left\{h=\left(\begin{array}{cc}\alpha & \beta \\ 0 & \alpha^{-1}\end{array}\right) \mid \alpha^{n}=1\right\}$ acts on the fiber $\mathcal{O}_{\Sigma_{n}}(-1)_{Q} \cong \mathbf{C}$ trivially. Thus $h$ acts on the fiber $E_{n}(a, b)_{Q} \cong$ $\mathbf{C}^{2}$ by multiplication by $\left(\begin{array}{cc}\alpha_{0}^{-b} & 0 \\ 0 & 1\end{array}\right)$. Hence for any point $P^{\prime} \in \mathbf{P}\left(E_{n}(a, b)\right)_{Q}, P^{\prime} \neq$ $(1,0),(0,1)$, we have $G_{P^{\prime}}=\left\{g \in B \mid \alpha^{n}=\alpha^{b}=1\right\}=F_{d}$, where $d=\operatorname{gcd}(n, b)$.

Now set $B_{i j}:=D_{i} \cap S_{j}(i=0, \infty, j=1,2)$ and consider the elementary transformation $\operatorname{elm}_{B_{i j}}$ of $X_{n}(a, b)$ with center $B_{i j}$, which is defined as the composition of the blowing-up along center $B_{i j}$ and the blowing-down of the proper transform of $D_{i}$.

Lemma 3.2. (1) $\operatorname{elm}_{B_{01}}\left(X_{n}(a, b)\right) \cong X_{n}(a-1, b-n)$.

(2) $\operatorname{elm}_{B_{02}}\left(X_{n}(a, b)\right) \cong X_{n}(a+1, b+n)$.

(3) $\operatorname{elm}_{B_{\infty 1}}\left(X_{n}(a, b)\right) \cong X_{n}(a-1, b)$.

(4) $\operatorname{elm}_{B_{\infty 2}}\left(X_{n}(a, b)\right) \cong X_{n}(a+1, b)$. 
Proof. We show (1) and the other assertions can be checked similarly. Let $E$ be the exceptional divisor of the blowing-up with center $B_{01}$. We denote by $\bar{E}$ (resp. $\bar{D}_{\infty}, \bar{S}_{j}$ ) the proper transform of $E$ (resp. $\left.D_{\infty}, S_{j}\right)$ in $\operatorname{elm}_{B_{01}}\left(X_{n}(a, b)\right)$. Then $\bar{S}_{1}$ and $\bar{S}_{2}$ are disjoint $G$-equivariant sections of the $\mathbf{P}^{1}$-bundle $\operatorname{elm}_{B_{01}}\left(X_{n}(a, b)\right) \rightarrow \Sigma_{n}$ and hence $\operatorname{elm}_{B_{01}}\left(X_{n}(a, b)\right)$ is the projectivization of a direct sum of two $G$-line bundles. Thus we may assume that $\operatorname{elm}_{B_{01}}\left(X_{n}(a, b)\right)$ is isomorphic to $X_{n}(c, d)$ for some $c, d \in \mathbf{Z}$.

Now we have $\bar{D}_{\infty} \cong \Sigma_{|b-n a|}, \bar{S}_{j} \cong \Sigma_{n}$ clearly, and $\bar{E} \cong \Sigma_{|b-n|}$ by Lemma 3.3 (1) below. If we set $l_{1}=\bar{E} \cap \bar{S}_{1}, l_{2}=\bar{S}_{1} \cap \bar{D}_{\infty}$, then $\left(l_{1}, l_{1}\right)_{\bar{E}}=b-n$ and $\left(l_{2}, l_{2}\right)_{\bar{D}_{\infty}}=b-n a$ by Lemma $3.3(2)$. Hence we have $(c, d)=(a-1, b-n)$ or $(1-a, n-b)$. Since $X_{n}(c, d) \cong X_{n}(-c,-d)$, we get the conclusion.

The following lemma is well-known and we omit the proof (cf. [U4, Lemma (8.5.4)]).

Lemma 3.3. Let $X$ be a smooth projective threefold and $S_{1}, S_{2} \subset X$ connected smooth surfaces intersecting transversally along a smooth connected curve $C$ isomorphic to $\mathbf{P}^{1}$. Suppose $(C, C)_{S_{i}}=a_{i}(i=1,2)$, where $(\cdot, \cdot)_{S_{i}}$ is the intersection form on $S_{i}$. Then the following assertions hold:

(1) $N_{X}(C) \cong \mathcal{O}_{\mathbf{P}^{1}}\left(a_{1}\right) \oplus \mathcal{O}_{\mathbf{P}^{1}}\left(a_{2}\right)$, where $N_{X}(C)$ is the normal bundle of $C$ in $X$. In particular, if we blow-up $X$ along $C$, then the exceptional divisor $E$ is isomorphic to $\mathbf{P}\left(\mathcal{O}_{\mathbf{P}^{1}}\left(-a_{1}\right) \oplus \mathcal{O}_{\mathbf{P}^{1}}\left(-a_{2}\right)\right) \cong \Sigma_{\left|a_{1}-a_{2}\right|}$.

(2) Let $\bar{X}$ be the blowing-up of $X$ along $C, \bar{S}_{i}$ the proper transform of $S_{i}$ in $\bar{X}$. Set $l_{i}=\bar{S}_{i} \cap E$. Then we have $\left(l_{1}, l_{1}\right)_{E}=a_{2}-a_{1}$ and $\left(l_{2}, l_{2}\right)_{E}=a_{1}-a_{2}$.

3.2. $Y(L, n) \in \mathcal{C}\left(F_{n}\right)$. Let $C$ be a smooth projective curve with the trivial $G$-action and $L$ a line bundle on $C$ with the trivial $G$-linearization. Consider $C \times \mathbf{P}\left(V_{2}\right) \cong$ $C \times \mathbf{P}^{1}$ with the product $G$-action and let $p_{1}: C \times \mathbf{P}^{1} \rightarrow C\left(\right.$ resp. $\left.p_{2}: C \times \mathbf{P}^{1} \rightarrow \mathbf{P}^{1}\right)$ be the projection to the first (resp. second) factor. For a positive integer $n$, we set $Y(L, n):=\mathbf{P}\left(p_{1}^{*}(L) \otimes p_{2}^{*}\left(\mathcal{O}_{\mathbf{P}^{1}}(-n)\right) \oplus \mathcal{O}_{C \times \mathbf{P}^{1}}\right)$. Let $r: Y(L, n) \rightarrow C \times \mathbf{P}^{1}$ be the projection and set $r_{1}=p_{1} \circ r$. Then, for any $x \in C$, we have $r_{1}^{-1}(x) \cong \Sigma_{n}$ and hence $Y(L, n) \in \mathcal{C}\left(F_{n}\right)$. We denote by $S_{\infty}$ (resp. $S_{0}$ ) the $G$-section of $r$ defined by the projection $p_{1}^{*}(L) \otimes p_{2}^{*}\left(\mathcal{O}_{\mathbf{P}^{1}}(-n)\right) \oplus \mathcal{O}_{C \times \mathbf{P}^{1}} \rightarrow p_{1}^{*}(L) \otimes p_{2}^{*}\left(\mathcal{O}_{\mathbf{P}^{1}}(-n)\right)$ (resp. $\left.p_{1}^{*}(L) \otimes p_{2}^{*}\left(\mathcal{O}_{\mathbf{P}^{1}}(-n)\right) \oplus \mathcal{O}_{C \times \mathbf{P}^{1}} \rightarrow \mathcal{O}_{C \times \mathbf{P}^{1}}\right)$.

We consider the elementary transformation $\operatorname{elm}_{s_{0}}\left(\right.$ resp. $\left.\operatorname{elm}_{s_{\infty}}\right)$ of $Y(L, n)$ with center $s_{0}:=S_{0} \cap r_{1}^{-1}(x)$ (resp. $s_{\infty}:=S_{\infty} \cap r_{1}^{-1}(x)$ ), which is defined as the composition of the blowing-up of $s_{0}$ (resp. $s_{\infty}$ ) and the blowing-down of the proper transform of $r_{1}^{-1}(x)$. The following lemma is checked by a similar method as in Lemma 3.2.

Lemma 3.4. The $\mathbf{P}^{1}$-bundle $\operatorname{elm}_{s_{0}}(Y(L, n))$ is isomorphic to $Y\left(L^{\prime}, n\right)$ for some $L^{\prime} \in \operatorname{Pic}(C)$. The same assertion holds for $\operatorname{elm}_{s_{\infty}}(Y(L, n))$.

3.3. $Z(m, n) \in \mathcal{C}\left(F_{d}\right)$, where $d=\operatorname{gcd}(m, n)$. For given two integers $m, n$ with $(m, n) \neq(0,0)$, set $E(m, n):=\mathcal{O}_{\mathbf{P}^{1}} \oplus \mathcal{O}_{\mathbf{P}^{1}}(m) \oplus \mathcal{O}_{\mathbf{P}^{1}}(n)$, which is a $G$-vector bundle over $\mathbf{P}\left(V_{2}\right) \cong \mathbf{P}^{1}$. We projectivize $E(m, n)$ to get a $\mathbf{P}^{2}$-bundle $Z(m, n):=$ $\mathbf{P}(E(m, n))$ with the induced $G$-action. We denote by $r_{m, n}: Z(m, n) \rightarrow \mathbf{P}^{1}$ the projection.

Lemma 3.5. $Z(m, n) \in \mathcal{C}\left(F_{d}\right)$, where $d=\operatorname{gcd}(m, n)$. 
Proof. Take a point $P=(1,0) \in \mathbf{P}^{1} . g=\left(\begin{array}{cc}a & b \\ 0 & a^{-1}\end{array}\right) \in G_{P}=B$ acts on the fiber $E(m, n)_{P} \cong \mathbf{C}^{3}$ by multiplication by

$$
\left(\begin{array}{ccc}
1 & 0 & 0 \\
0 & a^{-m} & 0 \\
0 & 0 & a^{-n}
\end{array}\right)
$$

Hence for a general point $Q \in \mathbf{P}(E(m, n))_{P} \cong \mathbf{P}^{2}$, we have $G_{Q}=\left\{\left(\begin{array}{cc}a & b \\ 0 & a^{-1}\end{array}\right) \mid a^{m}=\right.$ $\left.a^{n}=1\right\}=F_{d}$, where $d=\operatorname{gcd}(m, n)$.

3.4. $W_{2 m} \in \mathcal{C}(N(T))(m \geq 0)$. We consider $\mathbf{P}\left(V_{3}\right) \cong \mathbf{P}^{2}$ and set

$$
W_{2 m}:=\mathbf{P}\left(\mathcal{O}_{\mathbf{P}^{2}} \oplus \mathcal{O}_{\mathbf{P}^{2}}(-2 m)\right) \text { for } m \geq 0 \text {. }
$$

We denote by $\rho_{2 m}$ the projection $W_{2 m} \rightarrow \mathbf{P}^{2}$.

Lemma 3.6. $W_{2 m} \in \mathcal{C}(N(T))$.

Proof. The $G$-action on $\mathbf{P}^{2} \cong \mathbf{P}\left(V_{3}\right)$ is given by $\alpha: \mathbf{S L}(2) \rightarrow \mathbf{P G L}(3)$ defined by

$$
\alpha\left(\left(\begin{array}{ll}
a & b \\
c & d
\end{array}\right)\right)=\left(\begin{array}{ccc}
a^{2} & 2 a b & b^{2} \\
a c & a d+b c & b d \\
c^{2} & 2 c d & d^{2}
\end{array}\right) .
$$

$\mathbf{P}^{2}$ consists of two orbits: $C=\left\{x_{1}^{2}-4 x_{0} x_{2}=0\right\} \cong G / B$ and $\mathbf{P}^{2}-C$ $\cong G / N(T)$. Take a point $P=(0,1,0) \in \mathbf{P}^{2}-C$. Then $G_{P}=N(T)$ acts on the fiber $\mathcal{O}_{\mathbf{P}^{2}}(-1)_{P} \cong \mathbf{C}$ by multiplication by $a d+b c= \pm 1$ since $\mathcal{O}_{\mathbf{P}^{2}}(-1)$ is the universal subbundle $\subset \mathbf{P}^{2} \times \mathbf{C}^{3}$. Hence $G_{P}=N(T)$ acts on the fiber $\left(\mathcal{O}_{\mathbf{P}^{2}} \oplus \mathcal{O}_{\mathbf{P}^{2}}(-2 m)\right)_{P} \cong \mathbf{C}^{2}$ trivially. Thus we conclude that any orbit through a point $\in \mathbf{P}\left(\mathcal{O}_{\mathbf{P}^{2}} \oplus \mathcal{O}_{\mathbf{P}^{2}}(-2)\right)_{P}$ is isomorphic to $G / N(T)$ and $W_{2 m} \in \mathcal{C}(N(T))$.

Let $S_{\infty}$ (resp. $S_{0}$ ) be the section of $\rho_{2 m}: W_{2 m} \rightarrow \mathbf{P}^{2}$ corresponding to the projection $\mathcal{O}_{\mathbf{P}^{2}} \oplus \mathcal{O}_{\mathbf{P}^{2}}(-2 m) \rightarrow \mathcal{O}_{\mathbf{P}^{2}}(-2 m)\left(\right.$ resp. $\left.\mathcal{O}_{\mathbf{P}^{2}} \oplus \mathcal{O}_{\mathbf{P}^{2}}(-2 m) \rightarrow \mathcal{O}_{\mathbf{P}^{2}}\right)$ and $D_{v}=\rho_{2 m}^{-1}(C) \cong \Sigma_{4 m}$. We consider the elementary transformation with center $s_{\infty}:=S_{\infty} \cap D_{v}$ and $s_{0}:=S_{0} \cap D_{v}$. Namely, we blow-up $W_{2 m}$ along $s_{\infty}$ (resp. $s_{0}$ ) and blow-down the proper transform of $D_{v}$ to get elm $s_{\infty}\left(W_{2 m}\right)\left(\right.$ resp. $\left.\operatorname{elm}_{s_{0}}\left(W_{2 m}\right)\right)$, which is a $\mathbf{P}^{1}$-bundle over $\mathbf{P}^{2}$. In the $m=0$ case, we consider $\operatorname{lm}_{s}\left(W_{0}\right)$, where $s$ is any $G$-equivariant section of $D_{v} \rightarrow C$. The proof of the following lemma is similar to that of Lemma 3.2 .

Lemma 3.7. (1) $\operatorname{elm}_{s_{0}}\left(W_{2 m}\right) \cong W_{2(m-1)}(m>0)$.

(2) $\operatorname{elm}_{s_{\infty}}\left(W_{2 m}\right) \cong W_{2(m+1)}$.

3.5. We note that $X_{n}(a, b)$ may be non relatively minimal or may have two different $\mathbf{P}^{1}$-bundle structures. In order to clarify this point, we calculate the closed cone $\overline{N E}(X)$ of effective curves on $X=X_{n}(a, b)$ following [Mo, Chapter 1].

In the following lemma, we can assume $a \leq 0$ since $X_{n}(a, b) \cong X_{n}(-a,-b)$. We also assume $n \geq 2$. Set $l_{i}=S_{i} \cap D_{\infty}(i=1,2), l_{3} \subset S_{1} \cong \Sigma_{n}$ a fiber of this ruled surface and $l_{4}$ a fiber of $p=p_{n}: X=X_{n}(a, b) \rightarrow \Sigma_{n}$.

Lemma 3.8. (1) If $b-n a<0$, then $\overline{N E}(X)=\mathbf{R}_{+}\left[l_{1}\right]+\mathbf{R}_{+}\left[l_{3}\right]+\mathbf{R}_{+}\left[l_{4}\right]$. If $b-n a \geq 0$, then $\overline{N E}(X)=\mathbf{R}_{+}\left[l_{2}\right]+\mathbf{R}_{+}\left[l_{3}\right]+\mathbf{R}_{+}\left[l_{4}\right]$.

(2) Let $K_{X}$ be the canonical bundle of $X$. Then we have $\left(K_{X}, l_{1}\right)=n a-b+n-2$ in the case $b-n a<0,\left(K_{X}, l_{2}\right)=b-n a+n-2$ in the case $b-n a \geq 0$ and $\left(K_{X}, l_{3}\right)=-a-2$. In particular, $\mathbf{R}_{+}\left[l_{3}\right]$ is an extremal ray $\left(i . e .,\left(K_{X}, l_{3}\right)<0\right)$ if and only if $a=0,-1 . \mathbf{R}_{+}\left[l_{1}\right]$ and $\mathbf{R}_{+}\left[l_{2}\right]$ are not extremal rays. 
(3) If $a=0$, then the contraction map $f_{R}$ of $=\mathbf{R}_{+}\left[l_{3}\right]$ gives another $\mathbf{P}^{1}$-bundle structure $X=X_{n}(0, b) \rightarrow \Sigma_{|b|}$ to $X$. If $a=-1$, then $f_{R}$ is the blowing-down of $S_{1} \cong \Sigma_{n}$ in the fiber direction and gives $X \rightarrow Z(b,-n)$ if $b \leq 0$ and $X \rightarrow$ $Z(-b,-b-n)$ if $b>0$.

(4) $X_{n}(a, b)(n \geq 2)$ is not relatively minimal in the sense of Definition 2.6 if and only if $a=-1$.

Proof. (1) Suppose $b-n a<0$. Let $C \subset X$ be an irreducible curve. If $C \subset S_{1}$, then $C \equiv \alpha l_{1}+\beta l_{3}(\alpha, \beta \geq 0)$ in $S_{1}$, since $l_{1}$ is the negative section in $S_{1} \cong \Sigma_{n}$. Hence $C \equiv \alpha l_{1}+\beta l_{3}$ also in $X$.

If $C \not \subset S_{1}$, then $p_{*}(C) \equiv \alpha p_{*}\left(l_{1}\right)+\beta p_{*}\left(l_{3}\right)$ for some $\alpha, \beta \geq 0$ since $p_{*}\left(l_{3}\right)$ is a fiber and $p_{*}\left(l_{1}\right)$ is the negative section in $\Sigma_{n}$. Set $\gamma=\left(S_{1}, C\right) \geq 0$. Then we claim that $C \equiv \alpha l_{1}+\beta l_{3}+\delta l_{4}$, where $\delta=\gamma-\beta a+\alpha(n a-b) \geq 0$. Indeed, we have $\operatorname{Pic}(X)=\mathbf{Z O}_{X}\left(S_{1}\right) \oplus p^{*}\left(\operatorname{Pic}\left(\Sigma_{n}\right)\right)$. For any $L \in \operatorname{Pic}\left(\Sigma_{n}\right)$, we have $\left(C, p^{*}(L)\right)=$ $\left(p_{*}(C), L\right)=\left(\alpha p_{*}\left(l_{1}\right)+\beta p_{*}\left(l_{3}\right), L\right)=\left(\alpha l_{1}+\beta l_{3}+\delta l_{4}, p^{*}(L)\right)$ and $\left(\alpha l_{1}+\beta l_{3}+\right.$ $\left.\delta l_{4}, S_{1}\right)=\alpha\left(l_{1}, S_{1}\right)+\beta\left(l_{3}, S_{1}\right)+\delta\left(l_{4}, S_{1}\right)=\alpha(b-n a)+\beta a+\delta=\gamma=\left(C, S_{1}\right)$. Hence in any case, we have $C \equiv \alpha l_{1}+\beta l_{3}+\delta l_{4}$ for some $\alpha, \beta, \delta \geq 0$ so that $\overline{N E}(X)=\mathbf{R}_{+}\left[l_{1}\right]+\mathbf{R}_{+}\left[l_{3}\right]+\mathbf{R}_{+}\left[l_{4}\right]$. The $b-n a \geq 0$ case is similarly checked.

(2) Set $D:=p^{-1}\left(p_{*}\left(l_{3}\right)\right) \cong \Sigma_{-a}$. Then $\left(K_{X}, l_{3}\right)=\left(\left.K_{X}\right|_{D}, l_{3}\right)_{D}$, where $\left.K_{X}\right|_{D}$ is the restriction of the canonical bundle $K_{X}$ to $D$. By adjunction formula, we have $\left(\left.K_{X}\right|_{D}, l_{3}\right)_{D}=\left(K_{D}-\left.D\right|_{D}, l_{3}\right)_{D}=\left(K_{D}, l_{3}\right)_{D}=\left(-2 l_{3}-(2-a) l_{4}, l_{3}\right)_{D}=-a-2$ since $\left(l_{3}, l_{3}\right)_{D}=a$ and $\left(l_{4}, l_{3}\right)_{D}=1$.

Suppose $b-n a<0$. We have $\left(K_{X}, l_{1}\right)=\left(\left.K_{X}\right|_{D_{\infty}}, l_{1}\right)_{D_{\infty}}=\left(K_{D_{\infty}}, l_{1}\right)_{D_{\infty}}-$ $\left(\left.D_{\infty}\right|_{D_{\infty}}, l_{1}\right)_{D_{\infty}}$. Now $\left(K_{D_{\infty}}, l_{1}\right)_{D_{\infty}}=\left(-2 l_{1}-(n a-b+2) l_{4}, l_{1}\right)_{D_{\infty}}=(-2)(b-n a)-$ $(n a-b+2)$. On the other hand, $\left(\left.D_{\infty}\right|_{D_{\infty}}, l_{1}\right)_{D_{\infty}}=\left(D_{\infty}, D_{\infty}, S_{1}\right)=\left(l_{1}, l_{1}\right)_{S_{1}}=-n$. Hence we conclude $\left(K_{X}, l_{1}\right)=n a-b-2+n$.

We have $\left(K_{X}, l_{2}\right)=b-n a+n-2$ by a similar computation. The second assertion is easily checked.

(3) Assume $a=0$. Then

$$
\begin{aligned}
X_{n}(0, b) & =\mathbf{P}\left(\pi_{n}^{*}\left(\mathcal{O}_{\mathbf{P}^{1}}(b)\right) \oplus \mathcal{O}_{\Sigma_{n}}\right)=\mathbf{P}\left(\pi_{n}^{*}\left(\mathcal{O}_{\mathbf{P}^{1}}(b) \oplus \mathcal{O}_{\mathbf{P}^{1}}\right)\right) \\
& \cong \Sigma_{n} \times_{\mathbf{P}^{1}} \mathbf{P}\left(\mathcal{O}_{\mathbf{P}^{1}}(b) \oplus \mathcal{O}_{\mathbf{P}^{1}}\right)=\Sigma_{n} \times_{\mathbf{P}^{1}} \Sigma_{|b|}
\end{aligned}
$$

By taking the second projection $\operatorname{pr}_{2}: X_{n}(0, b) \cong \Sigma_{n} \times_{\mathbf{P}^{1}} \Sigma_{|b|} \rightarrow \Sigma_{|b|}$, we get $X_{n}(0, b) \cong X_{|b|}(0,-n) . l_{3}$ is a fiber of $\mathrm{pr}_{2}$ and hence $f_{R}$ coincides with $\mathrm{pr}_{2}$.

Assume $a=-1$. Set $L:=\mathcal{O}_{X}(1) \otimes p^{*}\left(\mathcal{O}_{\Sigma_{n}}(1)\right) \in \operatorname{Pic}(X)$, where $\mathcal{O}_{X}(1)=$ $\mathcal{O}_{X}\left(S_{1}\right)$. Then it is easy to see that the natural map $\left(\pi_{n} \circ p\right)^{*}\left(\pi_{n} \circ p\right)_{*}(L) \rightarrow L$ is surjective and $\left(\pi_{n} \circ p\right)_{*} L \cong \mathcal{O}_{\mathbf{P}^{1}} \oplus \mathcal{O}_{\mathbf{P}^{1}}(b) \oplus \mathcal{O}_{\mathbf{P}^{1}}(-n)$. Hence we have an induced morphism $F: X \rightarrow \mathbf{P}\left(\left(\pi_{n} \circ p\right)_{*} L\right) \cong Z(b,-n)$ if $b \leq 0, Z(-b,-b-n)$ if $b>0$. Now, the restriction of $F$ to $D=p^{-1} p_{*}\left(l_{3}\right) \cong \Sigma_{1}$ coincides with the blowing-down $\Sigma_{1} \rightarrow \mathbf{P}^{2}$ of the negative section, and hence $F$ is the blowing-down of $S_{1} \cong \Sigma_{n}$ in the fiber direction. Thus $f_{R}$ coincides with $F$.

(4) follows from (3).

Remark. According to Theorem 1.4, $X_{n}(a, b)$ is $G$-birational to $X_{d}(0,0) \cong \Sigma_{d} \times$ $\mathbf{P}^{1}$, where $d=\operatorname{gcd}(n, b)$. Using two different $\mathbf{P}^{1}$-fibrations $\Sigma_{n} \leftarrow X_{n}(0, b) \cong$ $X_{|b|}(0, n) \rightarrow \Sigma_{|b|}$, we can transform $X_{n}(a, b)$ to $X_{d}(0,0)$ by elementary transformations as follows:

We start from $X_{n}(a, b)$, assuming $b \geq 0$ for simplicity. 
(i) If $b<n$, then go to step (ii). If $b \geq n$, then $b=n q+b^{\prime}$, where $0 \leq b^{\prime}<n$. We use the elementary transformations of type (1) and (2) in Lemma 3.2 to transform $X_{n}(a, b)$ to $X_{n}\left(a^{\prime}, b^{\prime}\right)$, where $b^{\prime}<n$.

(ii) Then we perform the elementary transformations of type (3) and (4) to get $X_{n}\left(0, b^{\prime}\right)$ with $b^{\prime}<n$. Now we exchange the two fibrations to get $X_{b^{\prime}}(0, n)$.

(iii) We go to step (i) and continue.

(iv) By the Euclidean division algorithm, we eventually get $X_{d}(0,0)$.

As for the extremal rays on $Y(L, n), Z(m, n)$ and $W_{2 m}$, we have the following three lemmas. Since the proofs of these lemmas are similar to that of Lemma 3.8, we omit them.

Lemma 3.9. Let $Y=Y(L, n)$ and assume $n \geq 2$. Set $D=\left(p_{2} \circ r\right)^{-1}(y)\left(y \in \mathbf{P}^{1}\right)$, $l_{\infty}=D \cap S_{\infty}, l_{0}=D \cap S_{0}, l_{1}=r_{1}^{-1}(x) \cap S_{\infty}(x \in C)$ and $l_{2}=$ a fiber of $r: Y \rightarrow C \times \mathbf{P}^{1}$. Then the following assertions hold:

(1) $\overline{N E}(Y)=\mathbf{R}_{+}\left[l_{\infty}\right]+\mathbf{R}_{+}\left[l_{1}\right]+\mathbf{R}_{+}\left[l_{2}\right]$ if $d=\operatorname{deg}(L) \leq 0$ and $\overline{N E}(Y)=$ $\mathbf{R}_{+}\left[l_{0}\right]+\mathbf{R}_{+}\left[l_{1}\right]+\mathbf{R}_{+}\left[l_{2}\right]$ if $d>0$.

(2) Let $g$ be the genus of the curve $C$. Then we have $\left(K_{Y}, l_{\infty}\right)=2 g-2-d$, $\left(K_{Y}, l_{0}\right)=2 g-2+d$ and $\left(K_{Y}, l_{1}\right)=n-2$. In particular, $\mathbf{R}_{+}\left[l_{1}\right]$ is not an extremal ray. $\mathbf{R}_{+}\left[l_{\infty}\right]$ is an extremal ray if and only if $g=0$ and $d=0,-1$ in the case $d \leq 0$, and $\mathbf{R}_{+}\left[l_{0}\right]$ is an extremal ray if and only if $g=0$ and $d=1$ in the case $d>0$.

(3) Suppose $C=\mathbf{P}^{1}$ and $d=0$, i.e., $L \cong \mathcal{O}_{\mathbf{P}^{1}}$. Then $f_{R}$, where $R=\mathbf{R}_{+}\left[l_{\infty}\right]$, gives another $\mathbf{P}^{1}$-bundle structure $Y=Y\left(\mathcal{O}_{\mathbf{P}^{1}}, n\right) \rightarrow \Sigma_{n}$. This $\mathbf{P}^{1}$-bundle structure coincides with $Y=X_{n}(0,0) \rightarrow \Sigma_{n}$. Suppose $C=\mathbf{P}^{1}$ and $L \cong \mathcal{O}_{\mathbf{P}^{1}}(-1)$. Then $f_{R}$ is the blowing-down of $S_{\infty}$ and gives $Y \rightarrow Z(0,-n)$. Suppose $C=\mathbf{P}^{1}$ and $L \cong \mathcal{O}_{\mathbf{P}^{1}}(1)$. Then $f_{R^{\prime}}$, where $R^{\prime}=\mathbf{R}_{+}\left[l_{0}\right]$, is the blowing-down of $S_{0}$ and gives $Y \rightarrow Z(-n,-n)$.

(4) $Y(L, n)$ is not relatively minimal if and only if $C$ is rational and $L \cong$ $\mathcal{O}_{\mathbf{P}^{1}}( \pm 1)$.

Lemma 3.10. Let $Z=Z(m, n)$ with $0 \geq m \geq n,(m, n) \neq(0,0), d=\operatorname{gcd}(m, n) \geq$ 2. Denote by $S_{1}$ (resp. $\left.S_{2}\right) \subset Z=Z(m, n)$ the closed 2-dimensional $G$-stable subset defined by the projection $E(m, n) \rightarrow \mathcal{O}_{\mathbf{P}^{1}}(m) \oplus \mathcal{O}_{\mathbf{P}^{1}}(n)(\operatorname{resp} . E(m, n) \rightarrow$ $\left.\mathcal{O}_{\mathbf{P}^{1}} \oplus \mathcal{O}_{\mathbf{P}^{1}}(n)\right)$. Set $l_{1}=S_{1} \cap S_{2}$ and $l_{2}=$ a line in $r_{m, n}^{-1}(x) \cong \mathbf{P}^{2}\left(x \in \mathbf{P}^{1}\right)$. Then the following assertions hold:

(1) $\overline{N E}(Z)=\mathbf{R}_{+}\left[l_{1}\right]+\mathbf{R}_{+}\left[l_{2}\right]$.

(2) $\left(K_{Z}, l_{1}\right)=-4 n+3 m-2$. In particular, $\mathbf{R}_{+}\left[l_{1}\right]$ is not an extremal ray.

(3) $Z(m, n)$ is relatively minimal.

Lemma 3.11. Let $W=W_{2 n}(m \geq 0)$. Denote by $l_{1}$ a line in $S_{\infty} \cong \mathbf{P}^{2}$ and by $l_{2}$ a fiber of $\rho_{2 m}$. Then the following assertions hold:

(1) $\overline{N E}(W)=\mathbf{R}_{+}\left[l_{1}\right]+\mathbf{R}_{+}\left[l_{2}\right]$.

(2) $\left(K_{W}, l_{1}\right)=2 m-3$. In particular, $R:=\mathbf{R}_{+}\left[l_{1}\right]$ is an extremal ray if and only if $m=0,1$.

(3) If $m=0$, then $f_{R}$ is the second projection $W_{0}=\mathbf{P}\left(V_{3}\right) \times \mathbf{P}^{1} \rightarrow \mathbf{P}^{1}$. If $m=1$, then $f_{R}: W_{2} \rightarrow Y$ is a birational contraction collapsing $S_{\infty}$, and $Y$ is isomorphic to the Veronese cone in $\mathbf{P}\left(V_{5} \oplus V_{1}\right) \cong \mathbf{P}^{5}$.

(4) $W_{2 m}$ is relatively minimal. 


\section{Classification of Relatively minimal models}

In this section, we determine the structures of the relatively minimal models in $\mathcal{C}(H)$, where $H=F_{n}(n \geq 2)$ or $N(T)$. The results are summarized in the following two theorems:

Theorem 4.1. Let $X \in \mathcal{C}\left(F_{n}\right)(n \geq 2)$ be a relatively minimal model. Then $X$ is isomorphic to one of the following:

(1) $X_{n d}(a, n b)(a \leq 0, a \neq-1, \operatorname{gcd}(d, b)=1)$.

(2) $Y(L, n)\left(L \in \operatorname{Pic}(C), C:\right.$ non-rational, or $C \cong \mathbf{P}^{1}$ and $\left.L \nRightarrow \mathcal{O}_{\mathbf{P}^{1}}( \pm 1)\right)$.

(3) $Z(n s, n t)(0 \geq s \geq t,(s, t) \neq(0,0), \operatorname{gcd}(s, t)=1)$.

Theorem 4.2. Let $X \in \mathcal{C}(N(T))$ be a relatively minimal model and $C$ the invariant curve of $X$.

(1) If $C$ is rational, then $X \cong W_{2 m}$ for some $m \geq 0$.

(2) If $C$ is non-rational, then $X \cong \mathbf{P}\left(V_{3}\right) \times C$.

Remark. In Theorem 4.1, some relatively minimal models appear under different symbols. They are $X_{n}(0, b) \cong X_{n}(0,-b) \cong X_{|b|}(0, n)$ and $Y\left(\mathcal{O}_{\mathbf{P}^{1}}, n\right) \cong X_{n}(0,0)$.

Proof of Theorem 4.1. Let $X \in \mathcal{C}\left(F_{n}\right)(n \geq 2)$ be a relatively minimal model. Then there exists an extremal ray $R$ on $X$ and, since $X$ is relatively minimal, the contraction map $f_{R}$ is of fiber type by Proposition 2.2. Then our conclusion follows from Propositions 4.3, 4.5, 4.6 and 4.9 below.

Proposition 4.3. Let $X \in \mathcal{C}\left(F_{n}\right)(n \geq 2)$ and $R$ an extremal ray of $X$ such that $f=f_{R}: X \rightarrow \Sigma_{n d}$ is a $G$-conic bundle $(d \geq 1)$ (cf. Proposition 2.3). Then $X$ is $G$-isomorphic to $X_{n d}(a, n b)$ for some $a, b \in \mathbf{Z}$ such that $\operatorname{gcd}(d, b)=1$.

Proof. We discuss the $d=1$ case and the $d \geq 2$ case separately.

(1) Suppose $d=1$. We first note that every orbit $O(x)$ contained in $f^{-1}\left(\Sigma_{n}^{\prime}\right)$ is isomorphic to $G / F_{n}$. Indeed, $O(x)$ is isomorphic to $G / F_{N}$ for some $N \mid n$ since $f(O(x))=\Sigma_{n}^{\prime} \cong G / F_{n}$. A general orbit $G / F_{n}$ of $X$ deforms to $G / F_{N}$ and hence $n=$ $N$ by [Du1]. We claim $f^{-1}\left(\Sigma_{n}^{\prime}\right) \cong \Sigma_{n}^{\prime} \times \mathbf{P}^{1}$, where $G$ acts on $\mathbf{P}^{1}$ trivially and on $\Sigma_{n}^{\prime} \times$ $\mathbf{P}^{1}$ by the product action. Indeed, fix a point $y_{0} \in \Sigma_{n}^{\prime}$. Then the correspondence $f^{-1}\left(\Sigma_{n}^{\prime}\right) \ni x \mapsto\left(f(x), O(x) \cap f^{-1}\left(y_{0}\right)\right) \in \Sigma_{n}^{\prime} \times \mathbf{P}^{1}$ is a $G$-isomorphism. Hence we find that $X$ is $G$-birational to $\Sigma_{n} \times \mathbf{P}^{1}$ over $\Sigma_{n}$. Let $\Phi: X \rightarrow \Sigma_{n} \times \mathbf{P}^{1}$ be a $G$-birational map over $\Sigma_{n}$. We eliminate the indeterminacy locus of $\Phi$ as follows:

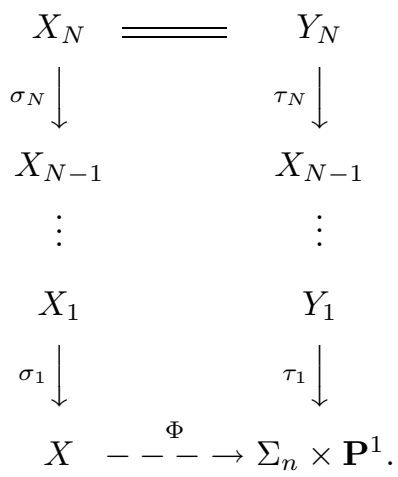

In the above diagram, $\Phi=\tau \circ \sigma^{-1}$, where $\sigma=\sigma_{1} \circ \sigma_{2} \circ \cdots \circ \sigma_{N}$ (resp. $\tau=$ $\left.\tau_{1} \circ \tau_{2} \circ \cdots \circ \tau_{N}\right), \sigma_{i}: X_{i} \rightarrow X_{i-1}$ (resp. $\tau_{j}: Y_{j} \rightarrow Y_{j-1}$ ) is a $G$-blowing-up along a smooth irreducible curve $B_{i-1} \subset X_{i-1}$ (resp. $\left.B_{j-1}^{\prime} \subset Y_{j-1}\right)$ isomorphic to $\mathbf{P}\left(V_{2}\right)$ 
$\left(X_{0}=X, X_{N}=Y_{N}, Y_{0}=\Sigma_{n} \times \mathbf{P}^{1}\right)$. Let $E_{N}:=\sigma_{N}^{-1}\left(B_{N-1}\right) \subset X_{N}$ be the exceptional divisor of $\sigma_{N}$. Then $E_{N}$ is $G$-isomorphic to either $\mathbf{P}_{\mathrm{tr}}^{1} \times \mathbf{P}\left(V_{2}\right)$ or $\Sigma_{l}$ $(l \geq 1)$ by deformation theory [Du1], where $\mathbf{P}_{\mathrm{tr}}^{1}=\mathbf{P}\left(V_{1}^{\oplus 2}\right)$. We note that $E_{N}$ is the proper transform of one of the following in $X_{N}=Y_{N}$ :

(i) the exceptional divisor $\tau_{j}^{-1}\left(B_{j-1}^{\prime}\right) \subset Y_{j}$,

(ii) $D_{0}=p_{1}^{-1}\left(C_{0}\right), D_{\infty}=p_{1}^{-1}\left(C_{\infty}\right) \subset Y_{0}=\Sigma_{n} \times \mathbf{P}^{1}$, where $p_{1}: \Sigma_{n} \times \mathbf{P}^{1} \rightarrow$ $\Sigma_{n}$ is the projection, since $E_{N}$ lies over $C_{0}$ or $C_{\infty} \subset \Sigma_{n}$. Consider the image $\tau\left(E_{N}\right) \subset \Sigma_{n} \times \mathbf{P}^{1}$. Assume that $\operatorname{dim} \tau\left(E_{N}\right)=1$. Then $\tau\left(E_{N}\right)$ is a section of $q_{0}=\left.p_{1}\right|_{D_{0}}: D_{0} \rightarrow C_{0}$ or $q_{\infty}=\left.p_{1}\right|_{D_{\infty}}: D_{\infty} \rightarrow C_{\infty}$, and $\left.\tau\right|_{E_{N}}: E_{N} \rightarrow \tau\left(E_{N}\right)$ coincides with $\mathbf{P}_{\mathrm{tr}}^{1} \times \mathbf{P}\left(V_{2}\right) \rightarrow \mathbf{P}\left(V_{2}\right)$ or $\Sigma_{l} \stackrel{\pi_{l}}{\rightarrow} \mathbf{P}^{1}$. Hence $\sigma_{N}$ is unnecessary and we can reduce $N$ by 1 .

Assume that $\operatorname{dim} \tau\left(E_{N}\right)=2$. Then $\tau\left(E_{N}\right)=D_{0}$ or $D_{\infty}$, which implies that $E_{N}$ is the proper transform of $D_{0}$ or $D_{\infty}$. We note that $\tau$ factors through the blowingup of $Y_{0}$ along $s$, where $s$ is a $G$-section of $q_{0}$ or $q_{\infty}$. Indeed, we have $\left(E_{N}, l_{1}\right)=-1$ and $\left(D_{0}, l_{2}\right)=\left(D_{\infty}, l_{\infty}\right)=0$, where $l_{1}$ is a fiber of $\left.\sigma_{N}\right|_{E_{N}}$ and $l_{2}$ (resp. $\left.l_{\infty}\right)$ is the fiber of $q_{0}$ (resp. $\left.q_{\infty}\right)$. Hence there exists an $i(1 \leq i \leq N)$ such that $\tau_{i}$ is the blowing-up with center $s$.

Now we perform the elementary transformation $\operatorname{elm}_{s}$. Then $\operatorname{elm}_{s} \circ \tau$ is a morphism and it collapses $l_{1}$ to a point. Hence $\sigma_{N}$ is unnecessary and we can reduce $N$ by 1 by replacing $Y=\Sigma_{n} \times \mathbf{P}^{1}$ by $X_{n}( \pm 1,0)$ or $X_{n}( \pm 1, \pm n)$ (cf. Lemma 3.2). Repeating this process, we find that $\Phi$ is a composition of finitely many elementary transformations and a $G$-isomorphism over $\Sigma_{n}$. Hence we conclude $X \cong X_{n}(a, n b)$ $(a, b \in \mathbf{Z})$.

(2) Suppose $d \geq 2$. Let $g_{d}: \Sigma_{n} \rightarrow \Sigma_{n d}$ be the cyclic covering of degree $d$ defined in Lemma 1.1 and form a fibered product $\bar{X}:=\Sigma_{n} \times_{\Sigma_{n d}} X$. Then a general orbit of $\bar{X}$ is $G$-isomorphic to $G / F_{n}$. Let $\bar{f}: \bar{X} \rightarrow \Sigma_{n}$ be the projection to the first factor. Then we have $\bar{f}^{-1}\left(\Sigma_{n}^{\prime}\right) \cong \Sigma_{n}^{\prime} \times \mathbf{P}^{1}$ as in the $d=1$ case. Hence we find that $f^{-1}\left(\Sigma_{n d}^{\prime}\right)$ is $G$-isomorphic to one of the $\mathbf{Z} /(d)$-quotient $Y$ of $\Sigma_{n}^{\prime} \times \mathbf{P}^{1}$ in Lemma 4.4 below. Thus $X$ is $G$-birational to $X_{n d}(0, m b)$ over $\Sigma_{n d}$, where $1 \leq b<d / 2$ with $\operatorname{gcd}(b, d)=1$ by Lemma $4.4(2)$. Then we find that $X$ can be obtained from $X_{n d}(0, n b)$ by elementary transformations in the same way as in (1) above. Hence $X$ is $G$-isomorphic to $X_{n d}(a, n b)$ for some $a, b \in \mathbf{Z}$ with $\operatorname{gcd}(d, b)=1$.

Lemma 4.4. Let $X:=\Sigma_{n}^{\prime} \times \mathbf{P}^{1}$ be the $G$-trivial fibration. Suppose that the cyclic group $\mathbf{Z} /(d)(d \geq 2)$ acts on $X G$-equivariantly in such a way that the quotient map $g_{d}^{\prime}: X \rightarrow Y$ is compatible with $g_{d}: \Sigma_{n}^{\prime} \rightarrow \Sigma_{n d}^{\prime}$ given in Lemma 1.1. Let $p_{1}^{\prime}: Y \rightarrow \Sigma_{n d}^{\prime}$ be the $G$-morphism induced by the projection $p_{1}: X \rightarrow \Sigma_{n}^{\prime}$. Further, assume that the general orbit of $Y$ is isomorphic to $G / F_{n}$.

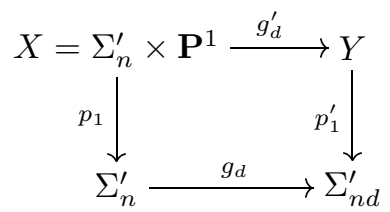

Then the following assertions hold:

(1) If $d>2$, then the number of different $G$-isomorphism classes of such $Y$ 's over $\Sigma_{n d}^{\prime}$ is $\varphi(d) / 2$, where $\varphi(d)$ is the number of integers $e(1 \leq e \leq d)$ such that $\operatorname{gcd}(e, d)=1$. If $d=2$, then there exists a unique $Y$ over $\Sigma_{n d}^{\prime}$. 
(2) Suppose $d>2$. Let $b_{i}(1 \leq i \leq \varphi(d) / 2)$ be an integer such that $\operatorname{gcd}\left(d, b_{i}\right)=1$ and $1=b_{1}<b_{2}<\ldots<b_{\varphi(d) / 2}<d / 2$. Then $p_{n d}: X_{n d}\left(0, n b_{i}\right) \rightarrow \Sigma_{n d}(1 \leq i \leq$ $\varphi(d) / 2)$ gives all the $Y$ 's over $\Sigma_{n d}^{\prime}$. If $d=2$, then $p_{2 n}: X_{2 n}(0, n) \rightarrow \Sigma_{n 2}$ is the unique $Y$ over $\Sigma_{2 n}^{\prime}$.

Proof. (1) We first note $\operatorname{Aut}^{G}\left(\Sigma_{n}^{\prime} \times \mathbf{P}^{1}\right) \cong \operatorname{Aut}^{G}\left(\Sigma_{n}^{\prime}\right) \times \operatorname{Aut}\left(\mathbf{P}^{1}\right) \cong \mathbf{C}^{\times} \times \mathbf{P S L}(2)$. We deal with the $d>2$ case. The $d=2$ case is similarly dealt with. Since the general orbit of $Y$ is isomorphic to $G / F_{n}$, we may assume that the $\mathbf{Z} /(d)$-action on $X$ is given by a subgroup $H_{\alpha}:=\left\langle\left(\zeta_{d}, \eta_{d}^{\alpha}\right)\right\rangle \subset \mathbf{C}^{\times} \times \mathbf{P S L}(2)$, where $\zeta_{d}:=e^{2 \pi \sqrt{-1} / d}$,

$$
\eta_{d}:=\left(\begin{array}{cc}
\zeta_{d} & 0 \\
0 & \zeta_{d}^{-1}
\end{array}\right) \in \mathbf{P S L}(2)
$$

if $d$ is odd and

$$
\eta_{d}:=\left(\begin{array}{cc}
\zeta_{2 d} & 0 \\
0 & \zeta_{2 d}^{-1}
\end{array}\right) \in \mathbf{P S L}(2)
$$

if $d$ is even, up to conjugation in $\operatorname{Aut}^{G}\left(\Sigma_{n}^{\prime} \times \mathbf{P}^{1}\right)$. Here $\alpha$ belongs to $I=\{1 \leq$ $\alpha<d / 2 \mid \operatorname{gcd}(d, \alpha)=1\}$. We note that the diagram in the statement is a fibered product, and $H_{\alpha}$ and $H_{\alpha^{\prime}}\left(\alpha, \alpha^{\prime} \in I, \alpha \neq \alpha^{\prime}\right)$ are not conjugate in $\mathbf{C}^{\times} \times \mathbf{P S L}(2)$. It follows that $X / H_{\alpha}(\alpha \in I)$ gives the $\varphi(d) / 2 Y^{\prime}$ 's over $\Sigma_{n d}^{\prime}$.

(2) We consider the $d>2$ case only since the $d=2$ case is similarly treated. For given integers $a, b, d, n$ with $d, n>0, \operatorname{gcd}(d, b)=1$, we construct a natural cyclic $G$-covering $\mu_{d}: X_{n}(d a, n b) \rightarrow X_{n d}(a, n b)$ of degree $d$ as follows: Let $g_{d}: \Sigma_{n} \rightarrow \Sigma_{n d}$ be the cyclic covering of degree $d$ given in Lemma 1.1. Since $g_{d}^{*}\left(E_{n d}(a, n b)\right) \cong$ $E_{n}(d a, n b)$ (see Subsection 3.1 for the notations), we define $\mu_{d}$ as

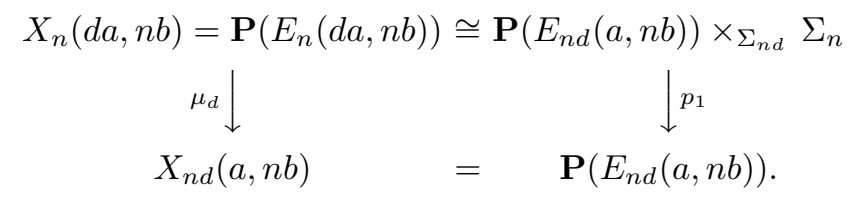

In particular, we have the following diagram setting $a=0, b=b_{i}$ :

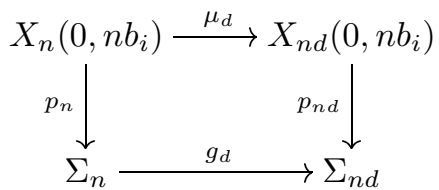

Now, we have $p_{n}^{-1}\left(\Sigma_{n}^{\prime}\right) \cong \Sigma_{n}^{\prime} \times \mathbf{P}^{1}$ and hence $p_{n d}^{-1}\left(\Sigma_{n d}^{\prime}\right)$ is isomorphic to one of the $Y$ 's over $\Sigma_{n d}^{\prime}$ in (1).

Assume that $X_{n d}\left(0, n b_{i}\right)$ is $G$-isomorphic to $X_{n d}\left(0, n b_{j}\right)$ over $\Sigma_{n d}^{\prime}$, and let $\Phi$ : $X_{n d}\left(0, n b_{i}\right) \rightarrow X_{n d}\left(0, n b_{j}\right)$ be a $G$-birational map over $\Sigma_{n d}$. Then by the same argument as in Proposition 4.3 (1), we find that $\Phi$ is a composition of finitely many elementary transformations and a $G$-isomorphism over $\Sigma_{m d}$. Hence we have $\pm n b_{i} \equiv n b_{j}(\bmod n d)$ by Lemma 3.2 , noting that $X_{n d}\left(0, n b_{i}\right) \cong X_{n d}\left(0,-n b_{i}\right)$. Since $1 \leq b_{i}, b_{j}<d / 2$, we conclude $b_{i}=b_{j}$.

Proposition 4.5. Let $X \in \mathcal{C}\left(F_{n}\right)(n \geq 2)$ and $R$ an extremal ray of $X$ such that $f_{R}: X \rightarrow C \times \mathbf{P}^{1}$ is a conic bundle. Then $X$ is $G$-isomorphic to $Y(L, n)$ for some $L \in \operatorname{Pic}(C)$. 
Proof. We first recall that $f_{R}$ is smooth and every fiber is isomorphic to $\mathbf{P}^{1}$ by Proposition 2.3.

We note that the invariant curve of $X$ is $C$. Indeed, let $C^{\prime}$ be the invariant curve of $X$. Since $k(C) \subset k\left(C^{\prime}\right), k\left(C^{\prime}\right)$ is a finite field extension of $k(C)$. On the other hand, $k(C)$ is algebraically closed in $k(X)$ and hence $k\left(C^{\prime}\right)=k(C)$.

Set $\varphi=\operatorname{pr}_{1} \circ f_{R}: X \rightarrow C$, where $\operatorname{pr}_{1}: C \times \mathbf{P}^{1} \rightarrow C$ is the projection to the first factor, and let $\rho: X \rightarrow \Sigma_{n} \times C$ be a $G$-birational map. Then $\rho$ induces an isomorphism $h: C \rightarrow C$ such that the following diagram commutes:

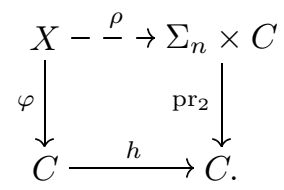

Then the same argument as in the proof of Proposition 4.3 (1) shows that $\rho$ is a composition of finitely many elementary transformations and a $G$-isomorphism over $C$. Thus we conclude that $X$ is isomorphic to $Y(L, n)$ for some $L \in \operatorname{Pic}(C)$ by Lemma 3.4.

Proposition 4.6. Let $X \in \mathcal{C}\left(F_{d}\right)(d \geq 2)$ and $R$ an extremal ray of $X$. Suppose that $f_{R}: X \rightarrow Y$ is a del Pezzo fiber space over a smooth projective curve $Y$. Then $X$ is isomorphic to $Z(a, b)$, where $a, b \in \mathbf{Z}, d=\operatorname{gcd}(a, b)$.

Proof. By Proposition 2.4, we know $Y \cong \mathbf{P}\left(V_{2}\right)$ and $f_{R}$ is a $\mathbf{P}^{2}$-bundle over $Y$. Then by Lemma 4.7 below, there exists a $G$-bundle $M$ on $Y$ of rank 3 such that $X$ is $G$-isomorphic to $\mathbf{P}(M)$. Take a point $P=(1,0) \in \mathbf{P}\left(V_{2}\right)$. Then $G_{P}=B$, and let $\alpha: B \rightarrow \mathbf{G L}(3, \mathbf{C})$ be the algebraic homomorphism which induces the $B$-action on the fiber $M_{P} \cong \mathbf{C}^{3}$. By Lemma 4.8 (2) below, we find that $\alpha$ is conjugate to $\Phi(a, b)$ of type (f) in Lemma $4.8(2)$. Since the $G$-bundle $M$ is determined by the $B$-action on $M_{P}$ (cf. [K, 6.3]), we find $M \otimes \mathcal{O}_{\mathbf{P}^{1}}(-l) \cong E(m-l, n-l)$ as $G$-bundles. Thus we conclude $X \cong Z(a, b)$, where $a=m-l, b-n-l$ and $\operatorname{gcd}(a, b)=1$.

Lemma 4.7. Let $E$ be a vector bundle of rank 3 on $\mathbf{P}\left(V_{2}\right) \cong \mathbf{P}^{1}$ and $Z:=\mathbf{P}(E)$. Suppose that $G$ acts on $Z$ in such a way that the projection $f: Z \rightarrow \mathbf{P}^{1}$ is $G$ equivariant. Then $E$ admits a $G$-linearization which induces the same $G$-action on $Z$ as the give one.

Proof. We first note that any line bundle $L \in \operatorname{Pic}(Z)$ is $G$-linearizable. Indeed, we have $\operatorname{Pic}(G \times Z) \cong \operatorname{Pic}(G) \times \operatorname{Pic}(Z) \cong \operatorname{Pic}(Z)$ since the irregularity of $Z$ is 0 and $\operatorname{Pic}(G) \cong\{0\}$. Hence there exists an isomorphism $\phi: \sigma^{*}(L) \rightarrow p_{2}^{*}(L)$, where $\sigma: G \times Z \rightarrow Z$ is a morphism defining the given $G$-action and $p_{2}: G \times Z$ is the projection to the second factor. Then we can modify $\phi$ so that it provides a $G$ linearization by the proof of [S, Theorem 1.6].

In particular, the tautological line bundle $\mathcal{O}_{\mathbf{P}(E)}(1)$ is $G$-linearizable and hence so is $E \cong r_{*}\left(\mathcal{O}_{\mathbf{P}(E)}(1)\right)$. Then the $G$-action on $\mathbf{P}(E)$ induced by this $G$-linearization coincides with the given one by construction. 
Lemma 4.8. (1) Let $\Phi: B=\mathbf{C}^{\times} \ltimes \mathbf{C} \rightarrow \mathbf{G L}(3)$ be an algebraic homomorphism. Then $\Phi(a, b)$ is equal to one of the following up to conjugation:
(a) $\left(\begin{array}{ccc}a^{n+2} & 0 & a^{n+2} b \\ 0 & a^{n+2} & a^{n+2} b \\ 0 & 0 & a^{n}\end{array}\right)$,
(b) $\left(\begin{array}{cc}a^{m+2} & a^{m+2} \\ 0 & a^{m} \\ 0 & 0\end{array}\right.$
$\left.\begin{array}{c}(1 / 2) a^{m+2} b^{2} \\ a^{m} b \\ a^{m-2}\end{array}\right)$
(c) $\left(\begin{array}{ccc}a^{l} & 0 & a^{l} b \\ 0 & a^{m} & 0 \\ 0 & 0 & a^{l-2}\end{array}\right)$,
(d) $\left(\begin{array}{ccc}a^{l} & 0 & 0 \\ 0 & a^{m} & 0 \\ 0 & 0 & a^{n}\end{array}\right)$.

(2) Consider the induced B-action on $\mathbf{P}^{2}$ by $\Phi(a, b)$ in (1). Then the general orbit in $\mathbf{P}^{2}$ is 1-dimensional if and only if $\Phi(a, b)$ is equal to one of the following:

(e) $\left(\begin{array}{ccc}a^{l} & 0 & a^{l} b \\ 0 & a^{l-2} & 0 \\ 0 & 0 & a^{l-2}\end{array}\right), \quad$ (f) $\left(\begin{array}{ccc}a^{l} & 0 & 0 \\ 0 & a^{m} & 0 \\ 0 & 0 & a^{n}\end{array}\right) \quad(l, m, n$ are not all equal).

If $\Phi$ is of type (e) (resp. type (f)), then the general orbit is isomorphic to $B / T$ (resp. $B / F_{d}$, where $\left.d=\operatorname{gcd}(l-m, l-n)\right)$.

Proof. (1) We may assume that $\operatorname{Im}(\Phi)$ is contained in the set of all upper triangular matrices. Let $\Phi_{1}: \mathbf{C}^{\times} \rightarrow \mathbf{G L}(3)$ be the restriction of $\Phi$ to $\mathbf{C}^{\times}$. Then up to conjugation, we may assume that

$$
\Phi_{1}(a)=\left(\begin{array}{ccc}
a^{l} & 0 & 0 \\
0 & a^{m} & 0 \\
0 & 0 & a^{n}
\end{array}\right) .
$$

Now, let $\Phi_{2}: \mathbf{C} \rightarrow \mathbf{G L}(3)$ be the restriction of $\Phi$ to $\mathbf{C}$. Then $\Phi_{2}(b)$ is of the form

$$
\left(\begin{array}{ccc}
1 & \phi_{12}(b) & \phi_{13}(b) \\
0 & 1 & \phi_{23}(b) \\
0 & 0 & 1
\end{array}\right)
$$

Since $\Phi_{2}$ is a homomorphism, $\phi_{12}(b)=h b, \phi_{23}(b)=k b$ for some $h, k \in \mathbf{C}$, and $\phi_{13}$ satisfies the following equation for any $b, B \in \mathbf{C}$ :

$$
\phi_{13}(b+B)=\phi_{13}(b)+\phi_{13}(B)+h k b B .
$$

It follows that $\phi_{13}(b)=p b+(h k / 2) b^{2}(p \in \mathbf{C})$.

Now, we have

$$
\Phi(a, b)=\Phi_{1}(a) \Phi_{2}(b)=\left(\begin{array}{ccc}
a^{l} & h a^{l} b & p a^{l} b+(h k / 2) a^{l} b^{2} \\
0 & a^{m} & k a^{m} b \\
0 & 0 & a^{n}
\end{array}\right) .
$$

By calculating the equation $\Phi((a, b) \cdot(A, B))=\Phi(a, b) \cdot \Phi(A, B)$ directly, we have the following three equations:

$$
\left\{\begin{array}{l}
\text { (i) } h a^{l} b\left(A^{m}-A^{l-2}\right)=0, \\
\text { (ii) } k a^{m} b\left(A^{n}-A^{m-2}\right)=0, \\
\text { (iii) } h k a^{l} b B\left(A^{m}-A^{l-2}\right)+(h k / 2) a^{l} b^{2}\left(A^{n}-A^{l-4}\right)+p a^{l} b\left(A^{n}-A^{l-2}\right)=0 .
\end{array}\right.
$$

From (i), we find that $h=0$ or $m=l-2$, and similarly for (ii), (iii). We may assume that $h=1$ (resp. $k=1$ ) if $h \neq 0$ (resp. $k \neq 0$ ) up to conjugation and thus finish the proof of (1). 
(2) In the case (a), set $P=(0,1,1) \in \mathbf{P}^{2}$. Then $B_{P}=\{( \pm 1,0)\} \subset B$. Hence the general orbit is 2-dimensional. In the case (b), we have $B_{P}=\{( \pm 1,0)\}$ for $P=(1,1,1)$, concluding that the general orbit is 2-dimensional. In the case (c), take a point $P=(0,1,1)$. Then $B_{P}=\left\{(a, 0) \mid a^{m}=a^{l-2}\right\}$. Hence if $m \neq l-2$, then the general orbit is 2-dimensional. Suppose that $m=l-2$. Then, for a general point $Q=(x, y, z), B_{Q}=\left\{(a, b) \mid a^{2}(x+b z)=x\right\} \cong T$. Hence the general orbit is 1-dimensional and isomorphic to $B / T$. In the case (d), for a general point $P=(x, y, z)$, we have $B_{P}=\left\{(a, b) \mid a^{l-m}=a^{l-n}=1\right\}$. If $l=m=n$, then $B$ acts on $\mathbf{P}^{2}$ trivially. Otherwise, the general orbit is 1-dimensional and isomorphic to $B / F_{d}$, where $d=\operatorname{gcd}(l-m, l-n)$.

Now let us turn to the $\rho(X)=1$ case. If $X \in \mathcal{C}\left(F_{n}\right)(n \geq 2)$ with $\rho(X)=1$, then $X$ is a Fano threefold of the first kind (i.e., $K_{X}^{-1}$ is ample and $\operatorname{Pic}(X) \cong \mathbf{Z}$ ).

Proposition 4.9. There does not exist a Fano threefold $X$ of the first kind such that $X \in \mathcal{C}\left(F_{n}\right)(n \geq 2)$.

Proof. Let $X \in \mathcal{C}\left(F_{n}\right)(n \geq 2)$ be a Fano threefold of the first kind. By Theorem 1.4, $X$ is $G$-birational to $Y:=\Sigma_{n} \times \mathbf{P}_{\mathrm{tr}}^{1}$. Let $p_{1}: Y \rightarrow \mathbf{P}_{\mathrm{tr}}^{1}$ (resp. $p_{2}: Y \rightarrow \Sigma_{n}$ ) be the projection to the first (resp. second) factor and set $S_{\infty}:=p_{2}^{-1}\left(C_{\infty}\right), S_{0}:=p_{2}^{-1}\left(C_{0}\right)$, $D_{x}:=p_{1}^{-1}(x) \cong \Sigma_{n}\left(x \in \mathbf{P}^{1}\right)$.

Let $\Phi: X \rightarrow Y$ be the $G$-equivariant birational map. We resolve the indeterminacy locus of $\Phi$ as usual; $\Phi=\tau \circ \sigma^{-1}, \sigma: X_{N+2} \rightarrow X$ is a composition of $G$-blowing-ups $\sigma_{i}: X_{i} \rightarrow X_{i-1}(1 \leq i \leq N+2)$ with center $B_{i-1} \cong \mathbf{P}\left(V_{2}\right)$, and $\tau: X_{N+2}=Y_{N} \rightarrow Y$ is a composition of $G$-blowing-ups $\tau_{j}: Y_{j} \rightarrow Y_{j-1}(1 \leq j \leq N)$ with center $C_{j-1} \cong \mathbf{P}\left(V_{2}\right)$. Let $E_{j} \subset Y_{j}$ be the exceptional divisor of $\tau_{j}$. Then $E_{j} \cong \mathbf{P}\left(V_{2}\right) \times \mathbf{P}_{\text {tr }}^{1}$ or $\Sigma_{l}(l \geq 1)$. Indeed, $E_{j}$ is a $\mathbf{P}^{1}$-bundle over $\mathbf{P}\left(V_{2}\right)$ and hence it is enough to see $E_{j} \nsucceq \mathbf{P}\left(V_{2}\right) \times \mathbf{P}\left(V_{2}\right)$. But this holds since the general fiber $G / F_{n}$ does not degenerate to $G / T$ by [Du1]. Now let $E_{i}^{\prime} \subset X_{i}$ be the exceptional divisor of $\sigma_{i}$. Then $E_{i}^{\prime}$ coincides with one of the proper transforms in $X_{i}$ of $S_{0}, S_{\infty}, D_{x}$ or $E_{j}$. Further, $\left.\sigma_{i}\right|_{E_{i}^{\prime}}: E_{i}^{\prime} \rightarrow B_{i-1}$ coincides with the unique $G$-equivariant projection $S_{0} \rightarrow \mathbf{P}\left(V_{2}\right), S_{\infty} \rightarrow \mathbf{P}\left(V_{2}\right), D_{x} \cong \Sigma_{n} \rightarrow \mathbf{P}\left(V_{2}\right)$ or $E_{j} \rightarrow \mathbf{P}\left(V_{2}\right)$. Hence we conclude that the rational map $\varphi:=\pi_{n} \circ p_{2} \circ \Phi: X \rightarrow \mathbf{P}\left(V_{2}\right) \cong \mathbf{P}^{1}$ is an everywhere defined $G$-morphism, where $\pi_{n}: \Sigma_{n} \rightarrow \mathbf{P}\left(V_{2}\right)$ is the projection.

Let $D:=\varphi^{-1}(z)\left(z \in \mathbf{P}^{1}\right)$. Then we have $D^{3}=0$, which is a contradiction since any effective divisor on a Fano threefold of the first kind is ample.

Finally we prove Theorem $4.2(\mathcal{C}(N(T))$-case $)$.

Proof of Theorem 4.2. (1) Let $\Phi: X \rightarrow W_{0}=\mathbf{P}\left(V_{3}\right) \times \mathbf{P}^{1}$ be a $G$-equivariant birational map and we resolve the indeterminacy locus of $\Phi$ as follows: $\Phi=\tau \circ \sigma^{-1}$, where $\sigma: X_{N} \rightarrow X$ is a composition of $G$-blowing-ups $\sigma_{i}: X_{i} \rightarrow X_{i-1}(1 \leq i \leq N)$, $X_{0}=X . \tau: X_{N}=W_{M} \rightarrow W_{0}$ is also a composition of $G$-blowing-ups $\tau_{j}: W_{j} \rightarrow$ $W_{j-1}(1 \leq j \leq M) . \sigma_{i}$ and $\tau_{j}$ are $G$-blowing-ups along a closed orbit isomorphic to $G / B \cong \mathbf{P}^{1}$.

Let $E_{N} \subset X_{N}$ be the exceptional divisor of $\sigma_{N}$. Then $E_{N}$ is isomorphic to either $\Sigma_{n}(n \geq 1)$ or $\mathbf{P}\left(V_{2}\right) \times \mathbf{P}_{\mathrm{tr}}^{1}$ since the general orbit $G / N(T)$ does not degenerate to $G / T$ by [Du1]. We consider the image of $E_{N}$ by $\tau$ which is a closed $G$-stable subset of $W_{0}$. The we have the following three cases (see Subsection 3.4 for the notations): (a) $\tau\left(E_{N}\right)=$ a section $s$ of $\left.\rho_{0}\right|_{D_{v}}: D_{v} \rightarrow C$. (b) $\tau\left(E_{N}\right)=D_{v} \cong \mathbf{P}\left(V_{2}\right) \times \mathbf{P}_{\mathrm{tr}}^{1}$. (c) $\tau\left(E_{N}\right)=$ a section of $\rho_{0} \cong \mathbf{P}\left(V_{3}\right)$. The case (c) clearly cannot occur. Suppose that 
(a) occurs. Then $\tau$ contracts the fiber of $\left.\sigma_{N}\right|_{E_{N}}$ to a point so that $\sigma_{N}$ is unnecessary and we may reduce $N$ by 1 . Suppose that (b) occurs. Then we can reduce $N$ by 1 by performing an elementary transformation. Thus $\Phi$ is a composition of elementary transformations of $W_{0}$ and a $G$-birational morphism. Since $X$ is relatively minimal, we conclude $X \cong W_{2 m}$ for some $m$ by Lemma 3.7 .

(2) Let $\Psi: X \rightarrow \mathbf{P}^{2} \times C$ be a $G$-equivariant birational map. We resolve the indeterminacy locus of $\Psi$ as in (1). Then $\tau\left(E_{N}\right)$ is a section of $D_{v} \rightarrow C$ and hence $\sigma_{N}$ is unnecessary. Repeating this process, we find that $X \cong \mathbf{P}\left(V_{3}\right) \times C$.

\section{APPENDIX}

In this appendix, we summarize the classification of $\mathcal{U S}(n)$ for $n=1,2,3$, where $\mathcal{U S}(n)$ is the set of isomorphism classes of smooth projective $n$-folds with unsolvable $\operatorname{Aut}^{0}(\cdot)$ as in Introduction.

Proposition A. (0) $X \in \mathcal{U S}(n)$ if and only if there exists a simple algebraic group $H$ such that $H$ acts on $X$ regularly and non-trivially.

In the following three assertions $H$ denotes a simple algebraic group.

(1) Let $X$ be a smooth projective $H$-curve. Then $H$ is isogenous to $\mathbf{S L}(2)$ and $X \cong \mathbf{P}^{1}$. Thus $\mathcal{U S}(1)$ consists of only $\mathbf{P}^{1}$.

(2) Let $X$ be a smooth projective $H$-surface. Then $H$ is isogenous to $\mathbf{S L}(3)$ or $\mathbf{S L}(2)$. If $H$ is isogenous to $\mathbf{S L}(3)$, then $X \cong \mathbf{P}^{2}$. If $H$ is isogenous to $\mathbf{S L}(2)$, then $X$ is isomorphic to $\mathbf{P}^{2}, \mathbf{P}^{1} \times \mathbf{P}^{1}, \Sigma_{n}(n \geq 1)$, or $C \times \mathbf{P}^{1}$, where $C$ is a smooth projective curve.

(3) Let $X$ be a smooth projective $H$-threefold. Then $H$ is isogenous to $\mathbf{S L}(i)$ $(2 \leq i \leq 4)$ or $\mathbf{S O}(5)$. If $H$ is isogenous to $\mathbf{S L}(4)$, then $X \cong \mathbf{P}^{3}$. If $H$ is isogenous to $\mathbf{S O}(5)$, then $X$ is isomorphic to either $\mathbf{P}^{3}$ or a smooth quadric threefold in $\mathbf{P}^{4}$. If $H$ is isogenous to $\mathbf{S L}(3)$, then $X$ is isomorphic to one of the following threefolds: $\mathbf{P}^{3}, \mathbf{P}\left(\mathcal{O}_{\mathbf{P}^{2}} \oplus \mathcal{O}_{\mathbf{P}^{2}}(-n)\right)(n \geq 0), \mathbf{P}\left(T_{\mathbf{P}^{2}}\right)$, or $C \times \mathbf{P}^{2}$, where $T_{\mathbf{P}^{2}}$ is the tangent bundle of $\mathbf{P}^{2}$ and $C$ is a smooth projective curve.

Proof. The assertion (0) follows from the structure theorem of algebraic groups (cf. $[\mathrm{R}$, Theorem 16]) and (1) is obvious. For the proofs of (2), (3), see [Mab, Theorem 5.1], [N1, Theorem 1], [N3, Propositions (1.1), (1.2)].

In the assertion (3) of Proposition A, the description of smooth projective SL(2)threefolds is omitted so that we give some references on this. Let $X$ be a smooth projective $\mathbf{S L}(2)$-threefold and $m$ the maximum dimension of all orbits of $X$. Suppose $m=3$ (i.e., $X$ has an open dense orbit). Then the birational geometry of such $X$ is completely clarified in [Mu], [U4] and [N2]. Suppose $m=1$. Then $X \cong S \times \mathbf{P}^{1}$, where $S$ is a smooth projective surface (this follows easily from [Mab, Theorem 1.3.1]). Thus, the $m=2$ case (i.e., $X$ has 2-dimensional general orbits) is the last class in $\mathcal{U S}(3)$ whose structure is unknown.

\section{REFERENCES}

[A] M. Artin, Algebraic approximation of structures over complete local rings, Inst. Hautes Etudes Sci. Publ. Math. 36 (1969), 23-58. MR 42:3087

[B] A. Bialynicki-Birula, On action of $\mathbf{S L}(2)$ on complete algebraic varieties, Pacific J. Math. 86 (1980), 53-58. MR 81i:14031

[CKM] H. Clemens, J. Kollár, and S. Mori, Higher dimensional complex geometry, Astérisque 166, Société Mathématique de France, 1988. MR 90j:14046 
[De] M. Demazure, Sous-groupes algébriques de rang maximum du groupe de Cremona, Ann. Sci. École Norm. Sup. (4e) 3 (1970), 507-588. MR 44:1672

[Du1] E. Duma, Algebraic SL(2)-actions: Deformations of infinite isotropy subgroups, Colloq. Math. 58 (1990), 221-231. MR 91g:14042

[Du2] _ On SL(2)-actions without 3-dimensional orbits, Colloq. Math. 58 (1990), 231241. MR 91f: 14043

[EF] F. Enriques and G. Fano, Sui gruppi di transformazioni cremoniane dello spazio, Ann. Mat. Pura Appl. (2 $\left.{ }^{a}\right) \mathbf{1 5}$ (1897), 59-98.

[F] G. Fano, I gruppi di Jonquiéres generalizzati, Atti della R. Accad. di Troino 33 (1898), 221-271.

[FG] W. Fischer and H. Grauert, Local-triviale Familien kompakter komplexer Mannigfaltigkeiten, Nachr. Akad. Wiss. Göttingen Math.-Phys. K1 II (1965), 89-94. MR 32:1731

[H] R. Hermann, Lie groups: History, frontiers and applications, Vol. 1 (Sophus Lie's 1880 Transformation Group Paper), Mat. Sci. Press, Brookline, 1975.

[K] H. Kraft, Algebraic automorphisms of affine space, Topological Methods in Algebraic Transformations Groups, H. Kraft et al. (eds.), Progress in Math. 80, Birkhäuser, Boston, Basel, Berlin, 1989, pp. 81-105. MR 91g:14044

[L] D. Luna, Slices étales, Bull. Soc. Math. France Mem. 33 (1973), 81-105. MR 49:7269

$[\mathrm{LV}] \quad$ D. Luna and Th. Vust, Plongements d'espaces homogènes, Comment Math. Helv. 58 (1983), 186-245. MR 85a:14035

[Mab] T. Mabuchi, On the classification of essentially effective $\mathbf{S L}(n, \mathbf{C})$-actions on algebraic $n$-folds, Osaka J. Math. 16 (1979), 745-759. MR 81k:14033b

[Mat] H. Matsumura, On algebraic groups of birational transformations, Rend. della Acad. Naz. del Lincei 34 (1963), 151-155. MR 28:3041

[Mo] S. Mori, Threefolds whose canonical bundles are not numerically effective, Ann. of Math. 116 (1982), 133-176. MR 84e:14032

$[\mathrm{Mu}]$ S. Mukai and H. Umemura, Minimal rational threefolds, Lecture Notes in Math., vol. 1016, M. Raynaud et al. (eds.), Springer-Verlag, Berlin, Heidelberg, New York, 1983, pp. 490-518. MR 85c: 14027

[N1] T. Nakano, Regular actions of simple algebraic groups on projective threefolds, Nagoya Math. J. 116 (1989), 139-148. MR 91b:14054

[N2] - On equivariant completions of 3-dimensional homogeneous spaces of $\mathbf{S L}(2, \mathbf{C})$, Japan. J. Math. 15 (1989), 221-273. MR 91b:14053

[N3] Regular actions of semi-simple algebraic groups on projective threefolds, especially $\mathbf{S L}(2)$, Group actions and invariant theory, A. Bialynicki-Birula et al. (eds.), CMS Conf. Proc. 10, AMS, Providence, Rhode Island, 1989, pp. 137-155. MR 90i:14051

[P] V. L. Popov, Quasi-homogeneous affine algebraic varieties of the group $\mathbf{S L}(2)$, Math. USSR Izv. 7 (1973), 793-831. MR 49:5018

[R] M. Rosenlicht, Some basic theorems on algebraic groups, Amer. J. Math. 78 (1956), 401443. MR 18:514a

[S] H. Sumihiro, Equivariant completion II, J. Math. Kyoto Univ. 15 (1975), 573-605. MR 52: 8137

[U1] H. Umemura, Sur les sous-groupes algébriques primitifs du groupe de Cremona à trois variables, Nagoya Math. J. 79 (1980), 47-67. MR 81k:14011

[U2] Maximal algebraic subgroups of the Cremona group of three variables (Imprimitive algebraic subgroups of exceptional type), Nagoya Math. J. 87 (1982), 59-78. MR 84b:14005

[U3] - On the maximal connected algebraic subgroups of the Cremona group II, Algebraic Groups and Related Topics, Advanced Studies in Pure Math. 6, Kinokuniya and NorthHolland, Tokyo, Amsterdam, New York, Oxford, 1985, pp. 349-436. MR 87d:14008

[U4] Minimal rational threefolds II, Nagoya Math. J. 110 (1988), 15-80. MR 89f: 14040

Department of Mathematical Sciences, College of Science and Engineering, Tokyo Denki University, Hatoyama-machi, Hiki-gun, Saitama-ken, 350-0394, Japan

E-mail address: nakano@r.dendai.ac.jp 